\title{
Evidence of nanostructure development from the molecular dynamics of poly(pentamethylene 2,5- furanoate)
}

Daniel E. Martínez-Tong ${ }^{1,2, *}$, Michelina Soccio ${ }^{3, *}$, Beatriz Robles-Hernández ${ }^{1,2}$, Giulia Guidotti $^{3}$, Massimo Gazzano ${ }^{4}$, Nadia Lotti ${ }^{3}$, Angel Alegria ${ }^{1,2}$

${ }^{1}$ Departamento de Polímeros y Materiales Avanzados: Física, Química y Tecnología. University of the Basque Country (UPV/EHU). Paseo Manuel Lardizábal 3, 20018 Donostia, Spain

${ }^{2}$ Centro de Física de Materiales (CFM, CSIC-UPV/EHU). Paseo Manuel Lardizábal 5, 20018 - Donostia, Spain.

${ }^{3}$ Civil, Chemical, Environmental and Materials Engineering Dept., University of Bologna, Via Terracini 28, 40131, Bologna, Italy

${ }^{4}$ Institute of Organic Synthesis and Photoreactivity -National Research Council, Via P. Gobetti, 101, 40129, Bologna, Italy

Corresponding authors: $\underline{\text { danielenrique.martinezt@ehu.eus }}$

m.soccio@unibo.it

\begin{abstract}
We report on the molecular origin of poly(pentamethylene 2,5-furanoate) particular physical properties. From the structural point of view we found this polymer can develop crystallinity when stored at room conditions for months. On the other hand, we used broadband dielectric spectroscopy (BDS) measurements, to analyze in very detail the local and segmental molecular dynamics of the material subjected to several thermal treatments. In this way we evidenced that the molecular dynamics are sensitive to thermal history over a broad temperature range. This behavior has been attributed to possible inter-chain interactions detected via infrared spectroscopy and rheology measurements in the non-crystallized polymer.
\end{abstract}




\section{Introduction}

Thanks to their lightness, versatility and affordable cost, plus the possibility to be appropriately modified for particular applications, plastic materials are widely produced and used. ${ }^{1}$ In particular, packaging is the industrial sector with the most significant use of plastics, and also the one in which the final products have the shortest shelf-life. ${ }^{1}$ That leads to the accumulation of large quantities of plastic waste in the environment that threatens both the terrestrial and marine habitat. ${ }^{2}$ In addition to heavy pollution, traditional plastics also lead to excessive use of non-renewable sources, especially oil. ${ }^{3}$

The strong academic and industrial commitment to the realization and implementation of new materials suitable to replace traditional plastics, and the current European Union directives, push towards the reuse of plastic products and their recycling. ${ }^{1,4-7}$ However, to meet the particular needs of a specific type of application, the polymeric matrix is often coupled with other compounds such as additives, paper, metals, as well as with other polymers. ${ }^{8-10}$ This multi-layered nature of a package generally hampers its recyclability. ${ }^{10-11}$ A possible solution to the problems of the so-wide use of plastics is constituted by biopolymers, i.e., materials that are produced from renewable sources and/or are biodegradable. ${ }^{3,}$ 12-13 Therefore, replacing traditional plastics with mono-material bioplastics is a desirable solution for a circular economy.

In this context, the scientific community's huge interest in the polyesters based on furandicarboxylic acid is not surprising, as evidenced by a large number of recent literature reports. ${ }^{14-25}$ The furandicarboxylic acid (FDCA) is a bifunctional aromatic monomer produced from biomass through an eco-sustainable process. ${ }^{26-32}$ It is mainly employed in the synthesis of polyesters ${ }^{14-25,33}$ and copolyesters with improved functional properties compared to their terephthalic acid-based counterparts, such as excellent thermal stability and processability, excellent barrier properties, and tunable mechanical 
response. ${ }^{17,34-52}$ For example, when compared with its terephthalic analog poly(ethylene terephthalate) (PET), poly(ethylene furanoate) (PEF) is easier to process due to its lower melting temperature and would perform better in applications because of its higher glass transition temperature. Moreover, PEF shows six times better barrier properties for $\mathrm{O}_{2}$ and three times for $\mathrm{CO}_{2}$ than PET, which makes it a very interesting material for the production of soft drink bottles. ${ }^{53-54}$ Some authors have highlighted how the properties of this family of polyesters can be suitably modulated by acting on the length of the glycol sub-chain. ${ }^{23,25}$ Specifically, the different number of methylene groups has a direct effect on the chain mobility and crystallizing capability. ${ }^{23,55-56}$ These characteristics allow to obtain from amorphous to semicrystalline polymers, which at room temperature can be in the glassy or in the rubbery state. According to these features, a different microstructure can develop, having a direct effect on the macroscopic properties. ${ }^{25}$

In the family of FDCA-based polyesters, poly(pentamethylene furanoate), PPeF, is attracting interest. ${ }^{24} \mathrm{PPeF}$ is characterized by a glass transition temperature below room temperature and shows crystallization kinetics so slow that, under normal conditions, it can be considered completely amorphous. ${ }^{24,55-56}$ Despite these characteristics, PPeF can be processed as films and, very surprisingly, it exhibits an elastic mechanical response at room temperature, as well as exceptional barrier properties. ${ }^{24,55}$ This behavior has been explained on the basis of particular inter-chain interactions, ${ }^{24}$ also proposed for the other polymers of the family. ${ }^{25}$ These interactions give rise to a microstructure responsible for the exceptional performances that make PPeF an excellent candidate for the realization of flexible mono-material devices, with outstanding gas barrier capabilities.

In this work, we have investigated the molecular origins of the peculiar and interesting properties of $\mathrm{PPeF}$, using a synergistic combination of different experimental techniques. Our results provide new insights on the study of this polymer and differentiate 
from previous publications, where the material was considered as fully amorphous ${ }^{23}$ and with physical properties independent from thermal history. ${ }^{56}$

\section{Experimental Section}

Samples. PPeF homopolymer has been synthesized according to the procedure described elsewhere and free-standing films have been prepared by compression molding as previously reported. ${ }^{25}$

Differential Scanning Calorimetry (DSC). DSC measurements were carried in a TA Instruments Q2000 equipment, with a liquid nitrogen cooling system. Heating/cooling ramps were conducted at a mean rate of $3 \mathrm{~K} / \mathrm{min}$. PPeF samples for DSC measurements were prepared by encapsulating c.a. $5 \mathrm{mg}$ of material in aluminum pans.

Atomic Force Microscopy (AFM). AFM experiments were carried out using a Bruker Multimode AFM, equipped with a Nanoscope V controller. Height images were captured using the PeakForce Tapping protocol and Tap150Al-G probes (BudgetSensors). The $512 \times 512$ pixel/lines images were taken at a $0.05 \mathrm{~V}$ PeakForce setpoint, and at a $0.3 \mathrm{~Hz}$ scan rate. For AFM imaging, we microtomed the PPeF films. In this way, we were able to study the material structure within its volume. The films were cut with a microtome that was equipped with a diamond knife at $0{ }^{\circ} \mathrm{C}$. A sample holder designed for crosssection analysis was used. This fixed the sample in the same position throughout the entire process (cutting + imaging).

X-ray diffracton (XRD). X-ray diffraction patterns were acquired with a X'Pert PRO diffractometer in reflection mode with copper $\mathrm{K}_{\alpha}$ radiation and a fast solid state $\mathrm{X}^{\prime}$ Celerator detector. The $2 \theta$ range $5-60^{\circ}$ was scanned in $0.1^{\circ}$ steps, counting $100 \mathrm{~s}$ /step. The crystallinity index $\left(\mathrm{X}_{\mathrm{c}}\right)$ was calculated from the $\mathrm{X}$-ray pattern as the ratio between the crystalline diffraction area $\left(A_{c}\right)$ and the whole area under the diffraction 
profile $\left(A_{t}\right)$, as $X_{c}=A_{c} / A_{t} \cdot 100 . A_{c}$ was obtained from the total area of the diffraction profile by subtracting the amorphous halo, which was modelled as a broad bell-shaped peak. The incoherent scattering was subtracted in advanced.

Broadband Dielectric Spectroscopy (BDS). BDS allows the study of molecular dynamics via the analysis of the complex dielectric permittivity, $\varepsilon^{*}(\omega)=\varepsilon^{\prime}(\omega)-$ $i \varepsilon^{\prime \prime}(\omega)$, where $\varepsilon^{\prime}(\omega)$ is the dielectric constant and $\varepsilon^{\prime \prime}(\omega)$ the dielectric losses. The complex dielectric permittivity is a function of the applied electric field frequency $(\omega=$ $2 \pi f$, being $f$ the frequency), and the temperature $T$. We performed BDS experiments in a frequency range $10^{-1} \leq f(\mathrm{~Hz}) \leq 10^{7}$, using a Novocontrol dielectric spectrometer, equipped with an Alpha dielectric interface. The temperature was controlled by a nitrogen jet (Quatro from Novocontrol), with a temperature stability during every single frequency sweep of $\pm 0.2 \mathrm{~K}$. PPeF samples for dielectric studies were prepared by melt-pressing, in order to obtain homogeneous films. These samples were pressed between two gold-plated disc electrodes of $40 \mathrm{~mm}$ diameter (lower electrode) and $20 \mathrm{~mm}$ diameter (upper electrode). We used narrow Teflon spacers (150 $\mu \mathrm{m}$ thick) to avoid possible shortcircuits.

The BDS data analysis was carried out using the Havriliak-Negami (HN) formalism, where the complex dielectric function can be described as ${ }^{57}$ :

$$
\varepsilon^{*}(\omega)=\varepsilon_{\infty}+\sum_{x} \Delta \varepsilon_{x}\left[1+\left(i \omega \tau_{\mathrm{HN}_{x}}\right)^{b_{x}}\right]^{-c_{x}}+\left(\frac{\sigma_{\mathrm{DC}}}{i \varepsilon_{0} \omega}\right)
$$

where $\Delta \varepsilon$ is the dielectric strength of the relaxation and $\tau_{\mathrm{HN}}$ a characteristic relaxation time; $b$ and $c$ are shape parameters related to the symmetric and asymmetric broadening, respectively. The summation in equation (1) extends over all the processes present in the experimental window at a specific temperature. Also, the last term in this equation 
accounts for the contribution of charge carriers to the dielectric signal, where $\sigma_{\mathrm{DC}}$ is the direct current (DC) conductivity and $\varepsilon_{0}$ the vacuum permittivity.

From $\tau_{\mathrm{HN}}$, the peak relaxation time $\left(\tau_{\max }\right)$ was calculated using the equation ${ }^{57}$ :

$$
\tau_{\mathrm{MAX}} \equiv \frac{1}{2 \pi f_{\mathrm{MAX}}}=\tau_{\mathrm{HN}}\left[\sin \frac{b \pi}{2+2 c}\right]^{-1 / b}\left[\sin \frac{b c \pi}{2+2 c}\right]^{1 / b}
$$

where $f_{\max }$ is the frequency of maximum loss, and the rest are the $\mathrm{HN}$-function parameters.

Rheology. Dynamic torsional shear experiments were conducted over the angular frequency range $10^{-2}-10^{2} \mathrm{rad} \cdot \mathrm{s}^{-1}$, using an ARES torsional rheometer from TA Instruments, under $\mathrm{N}_{2}$ atmosphere. Parallel plate geometry with $8 \mathrm{~mm}$ diameter tools was used. Sample thicknesses were about $0.5 \mathrm{~mm}$ and low strain amplitude was used to ensure linear regime.

Fourier Transform Infrared Spectroscopy (FTIR). FTIR spectra were taken using a Jasco 6300 FTIR spectrometer, working in transmission mode. The samples were deposited on a Zinc Selenide window from a solution of the polymer in chloroform. Prior measurement the samples were subjected to a high vacuum (pressure $<10^{-7} \mathrm{mbar}$ ) to allow residual solvent evaporation. The thermal treatments were performed inside the spectrometer, using a LinkamTHMS600 stage connected to a T95-LinkPad System controller.

\section{Results}

3.1. Thermal transitions and nanostructure development on PPeF. Figure 1 and Table 1 present the calorimetric traces and the corresponding DSC results, respectively, of heating ramps from 220 to $420 \mathrm{~K}$ of PPeF films subjected to three different thermal 
treatments: slow and fast cooling from high temperature and storage at room temperature for 18 months. In Figure 1, the continuous line is representative of the calorimetric response after cooling the polymer from 420 to $220 \mathrm{~K}$ at $0.5 \mathrm{~K} / \mathrm{min}$ (slow cooling). After this thermal treatment, PPeF showed no signs of crystallization/melting. As a matter of fact, we observed only the calorimetric step corresponding to the glass transition phenomenon $\left(T_{\mathrm{g}}\right)$ at $292 \mathrm{~K}$. In Figure 1, the DSC trace of a PPeF sample ballistically cooled (> $70 \mathrm{~K} / \mathrm{min}$ ) inside the DSC from 420 to $220 \mathrm{~K}$ (fast cooling) is represented by the dotted line. We observed that the subsequent heating run looked nearly identical to that of the slowly cooled sample, presenting the same values for $T_{\mathrm{g}}$ and $\Delta c_{\mathrm{p}}$ (Table 1 ). In particular, the $\Delta c_{\mathrm{p}}$ values in these two cases are close to that previously found by us for an amorphous poly(butylene 2,5-furanoate) (PBF) sample, ${ }^{58}$ and comparable to the ones reported by Guidotti et al. and Papamokos et al. for amorphous PPeF. ${ }^{23-24}$ The dash-dotted line in Figure 1 corresponds to the DSC trace of the sample stored in a desiccator, at room temperature, for 18 months (Aged-PPeF). This sample was ballistically cooled down from $300 \mathrm{~K}$ to $220 \mathrm{~K}$, in the DSC instrument, to avoid erasing its thermal history. During the subsequent heating scan, we observed a broader $T_{\mathrm{g}}$ step with inflection point at $289 \mathrm{~K}$, a value slightly lower compared to the slow and fast cooled samples, as well as a reduced $\Delta c_{\mathrm{p}}$. Also, we detected a multiple endothermic phenomenon, with maxima at $324 \mathrm{~K}$ and $337 \mathrm{~K}$, respectively. It must be noted that in previous works, bulk PPeF was considered as a fully amorphous polymer; however, the observed endothermic process has been ascribed to a melting phenomenon as confirmed below by means of other techniques too. In fact, in principle, both the $\Delta c_{\mathrm{p}}$ reduction as well as the presence of the endothermic peaks indicated the development of crystals during storage. The associated heat value is of the same order of magnitude as those previously found for other furan-based semicrystalline polymers ${ }^{23}$ on the contrary, the melting temperature is fairly lower. 
Table 1. DSC results of PPeF samples under different thermal treatments.

\begin{tabular}{|c|c|c|c|c|}
\hline Sample & $\boldsymbol{T}_{\mathbf{g}}(\mathbf{K})$ & $\Delta \boldsymbol{c}_{\mathbf{p}}(\mathbf{J} / \mathbf{g} \cdot \mathbf{K})$ & $\boldsymbol{T}_{\mathbf{m}}(\mathbf{K})$ & $\Delta \boldsymbol{H}_{\boldsymbol{m}}(\mathbf{J} / \mathbf{g})$ \\
\hline slow cooled-PPeF & $292 \pm 1$ & $0.37 \pm 0.01$ & --- & --- \\
\hline fast cooled-PPeF & $292 \pm 1$ & $0.37 \pm 0.01$ & --- & --- \\
\hline Aged-PPeF & $289 \pm 1$ & $0.27 \pm 0.02$ & $\begin{array}{l}324 \pm 1 \mathrm{~K} \\
337 \pm 1 \mathrm{~K}\end{array}$ & $23 \pm 1$ \\
\hline
\end{tabular}

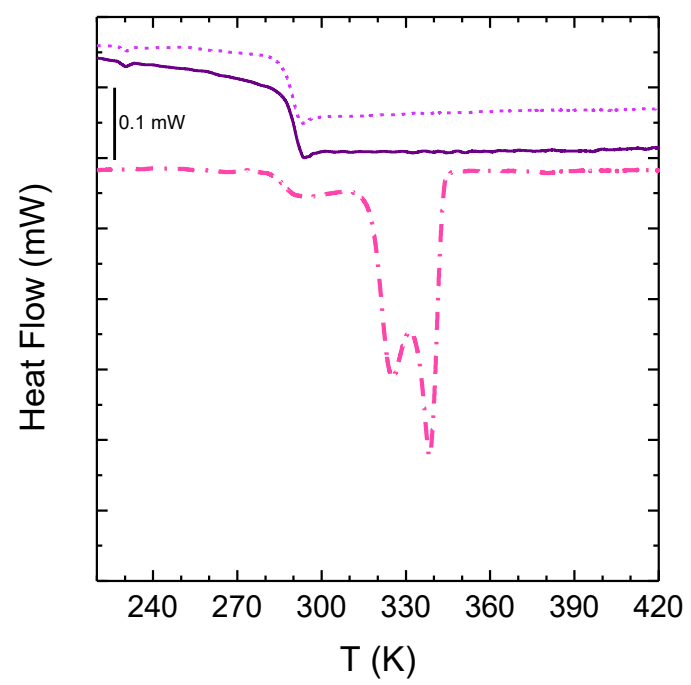

Figure 1. DSC curves (exo up) for PPeF samples undergoing different thermal histories: fast (dotted line) and slow cooling from the melt (solid line), storing for 18 months at room temperature (dash-dotted line).

To complement the DSC results, Figure 2 shows AFM height images of slow cooled and Aged-PPeF samples. In both cases, the height images were captured on the films cross-sections, as detailed in the experimental section. The AFM images of slow cooled PPeF, reported in Figures 2a-c, showed a flat surface with a mean roughness of about $1.3 \mathrm{~nm}$ (calculated using Figure 2a). The elongated topography features correspond to imperfections probably left by the microtoming process. Focusing the analysis onto a defect-free zone, as the image shown in Figure 2c, the mean roughness decreased down 
to $0.3 \mathrm{~nm}$. This value, similar to those reported for amorphous polymer films ${ }^{59}$, is in line with the DSC results (Figure 1, continuous line). Comparable results were found for a fast cooled PPeF sample (data not shown). Figures 2d-f show the AFM images for AgedPPeF. In this case, we observed a different topography. Now, there were randomly distributed nanostructures on the film's cross-section that did not cover the entire scanned area. The $10 \times 10 \mu \mathrm{m}$ image (Figure $2 \mathrm{~d}$ ) had a mean roughness of $3.4 \mathrm{~nm}$. This value only showed a small decrease, to $3.1 \mathrm{~nm}$, when calculated for the $2 \times 2 \mu \mathrm{m}$ image where most of the probed area is covered by the nanostructures (Figure $2 \mathrm{f}$ ). This represents a $10 \mathrm{x}$ increase in the mean roughness if comparing to the slow cooled sample, and it is comparable to those found for semicrystalline terephthalate-based polymer films ${ }^{60}$. Using Figure 2f, we studied the geometrical characteristics of the nanostructures. We found that the features had lengths in the $20-100 \mathrm{~nm}$ range and widths in the $10-20 \mathrm{~nm}$ range.
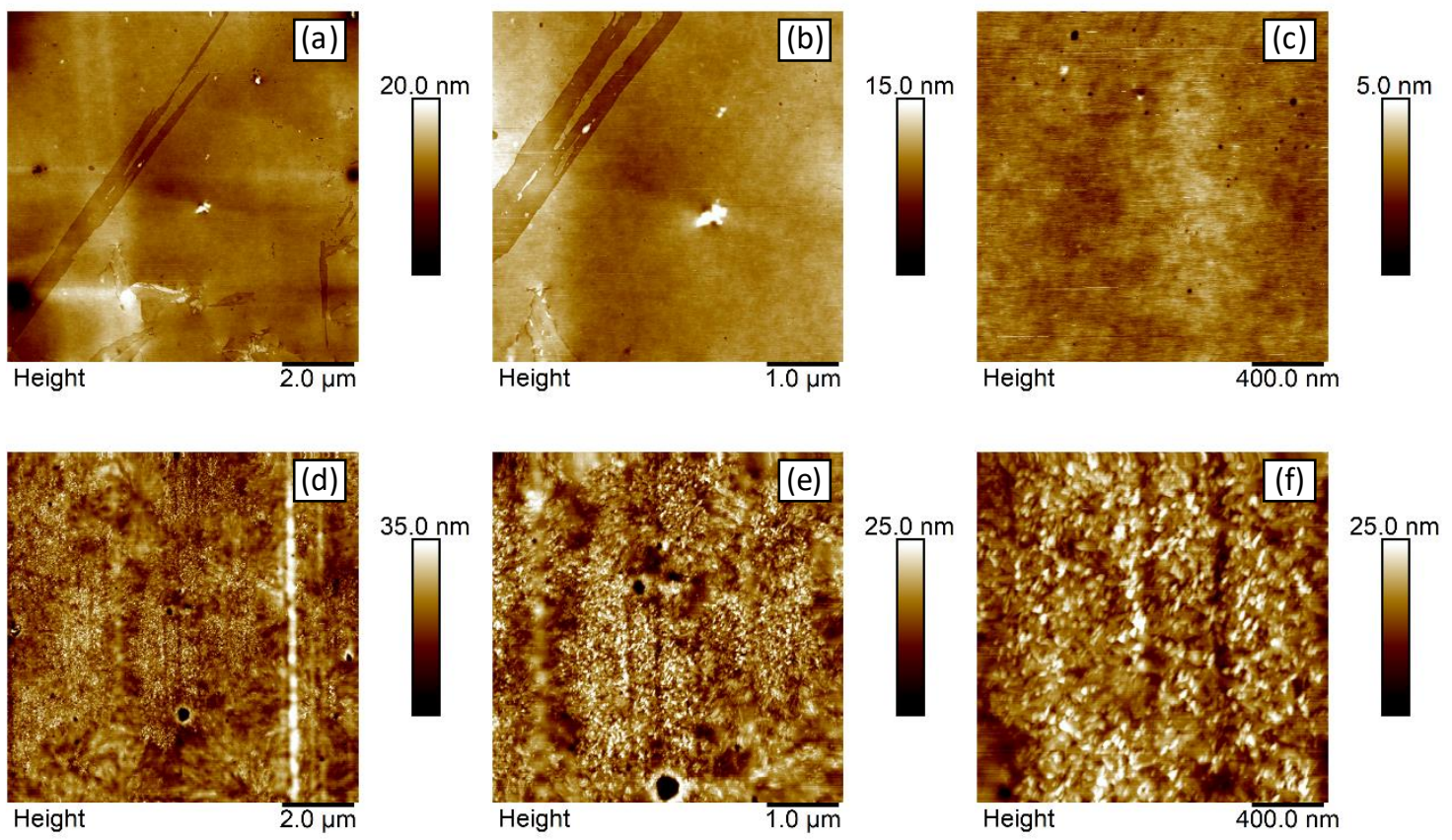

Figure 2. AFM height images of PPeF cross-sections: slow cooled PPeF (a-c) and aged-PPeF (d-f). Magnification increases from left to right. 
Lastly, Figure 3 shows the XRD patterns for slow cooled PPeF and Aged-PPeF. The slow cooled PPeF sample (dashed lines) showed the diffractometric pattern typical of an amorphous polymer sample, with amorphous halos, positioned at $q=1.48 \AA^{-1}(2 \theta$ $\left.=20.9^{\circ}\right)$ and $q=3.11 \AA^{-1}\left(44.6^{\circ}\right)$. The same results were found for the fast cooled PPeF sample (profile not shown). On the contrary, the profile of Aged-PPeF sample (solid line) was characterized by quite distinct features typical of a semi-crystalline material: several peaks overlapped to a bell-shaped background. The presence of a crystalline phase is coherent with the nanostructures detected through AFM analysis, and with the endothermic phenomena observed by DSC. The XRD peak positions are summarized in Table S1 (Supporting Information).

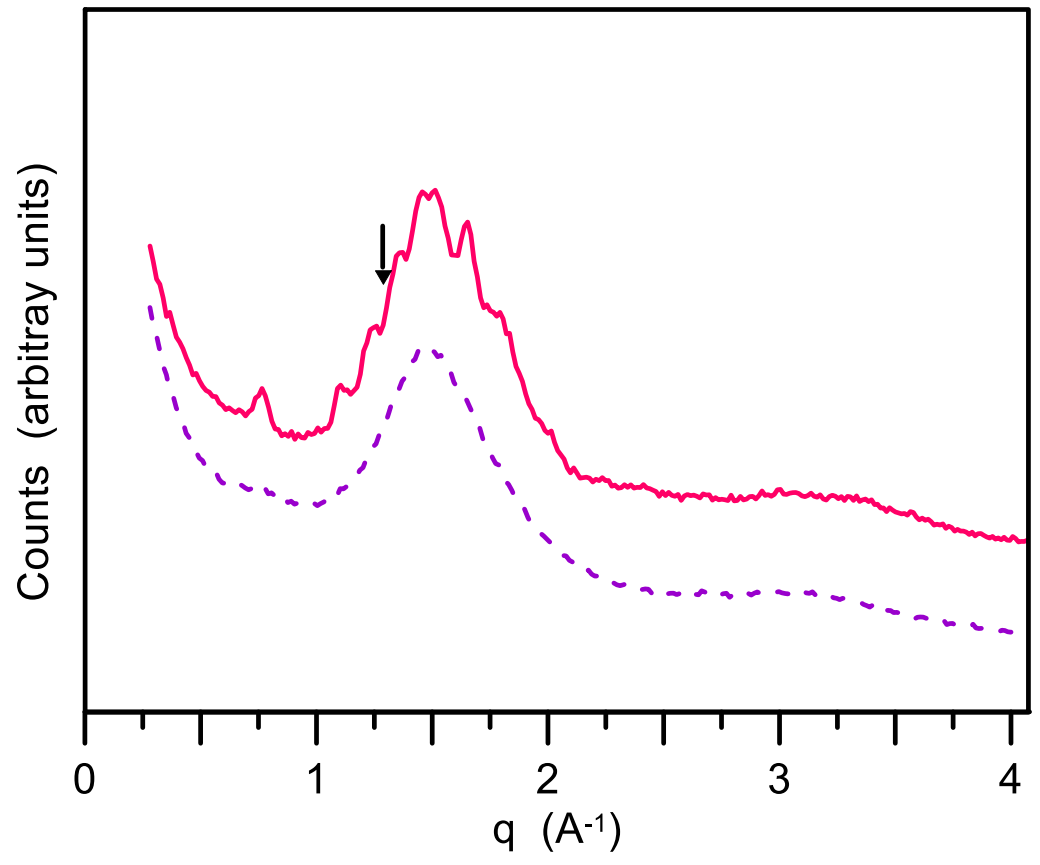

Figure 3. XRD results for slow cooled (dashed lines) and Aged-PPeF (solid lines) samples. Black arrow indicates the position of the maximum related to a mesophase, as reported by Guidotti et al., ${ }^{24}$ as presented in the discussion section of this work. The patterns are displaced in the vertical direction for clarity.

3.2. Impact of nanostructure formation on the PPeF molecular dynamics. We have studied, via BDS, the molecular dynamics of PPeF under different thermal protocols, as 
described in the following lines. Figure 4 shows the BDS results, as the imaginary part of the complex dielectric permittivity $\left(\varepsilon^{\prime \prime}\right)$ as a function of the electric field frequency. Empty triangles refer to the fast cooled PPeF sample (FC-PPeF). In this case, the film was heated inside the cryostat from room temperature up to $453 \mathrm{~K}$, held for $5 \mathrm{~min}$ and then the dielectric cell was immersed into a liquid $\mathrm{N}_{2}$ bath. Once the dielectric cell temperature was stable, it was transferred back to the cryostat, now at a temperature of $153 \mathrm{~K}$. Measurements started at $203 \mathrm{~K}$, heating in isothermal steps. Solid circles are relative to the results obtained for a sample measured in $5 \mathrm{~K}$ isothermal steps, cooling from $453 \mathrm{~K}$ to $203 \mathrm{~K}$ (SL-PPeF). Finally, crossed squares refer to the Aged-PPeF sample. In this case, the film was loaded into the dielectric cell at room temperature, and immediately cooled down to $203 \mathrm{~K}$ to start measuring in isothermal steps, heating up to $453 \mathrm{~K}$.

Local relaxations. Figure $4 \mathrm{a}$ shows the results at $243 \mathrm{~K}\left(T<T_{\mathrm{g}}\right)$. At this temperature, all the samples presented a broad relaxation, characterized by a single maximum around $10^{4} \mathrm{~Hz}$. The intensity and the asymmetry of the curves were both dependent on thermal history. Specifically, FC-PPeF was the sample with the highest intensity and higher asymmetry. Then, SL- and Aged-PPeF samples showed a progressive decrease in intensity and a more symmetric peak shape. To model these results, we used Cole-Cole (CC) functions, derived from eqn (1) by imposing $c_{x}=1$, and $\sigma_{\mathrm{DC}}=0$ $\mathrm{S} / \mathrm{cm}$, plus a power-law at low frequencies to account for upcoming relaxations (for further details, refer to Figure S2). For samples showing a nearly symmetrical local relaxation, SL- and Aged-PPeF, we used a single CC function. In the case of the FC-PPeF sample, the experimental data were fitted using a sum of two Cole-Cole functions to account for its asymmetry towards low frequencies. The faster component was called $\beta_{1}$, and the slower one $\beta_{2}$, in line with our previous report on poly(alkylene 2,5-furanoate)s' 
molecular dynamics. ${ }^{56,58}$ Lines in Figure 4a are representative fitting curves. In Figure 5, we show the temperature dependence of the resulting fitting parameters, for all three samples.
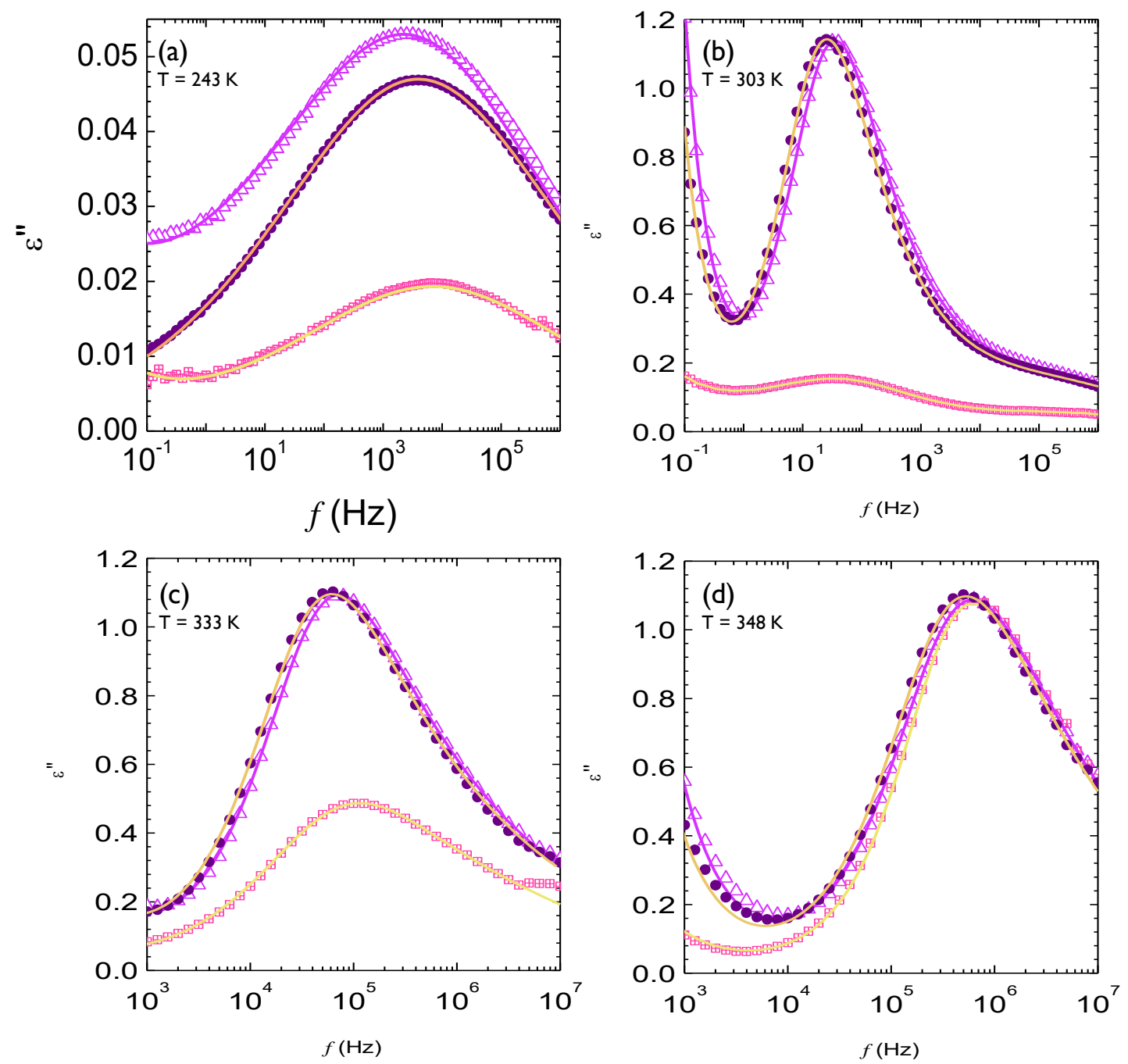

Figure 4. BDS results for FC-PPeF $(\triangle), \operatorname{SL}-\mathrm{PPeF}(\bullet)$ and Aged-PPeF ( $₫)$ at different temperatures: (a) $243 \mathrm{~K}$, (b) $303 \mathrm{~K}$, (c) $333 \mathrm{~K}$, (d) $348 \mathrm{~K}$. Continuous lines correspond to the total fits, as detailed in the text.

Figure 5a presents the Arrhenius relaxation map, where the local relaxations are located in the range $3.5 \leq 1000 / \mathrm{T}\left(\mathrm{K}^{-1}\right) \leq 5.0$. The temperature dependence of the local relaxations were well described using the Arrhenius equation: ${ }^{57}$ 


$$
\tau_{\mathrm{MAX}}(T)=\tau_{0} \exp \left[\frac{E_{A}}{R T}\right]
$$

where $\tau_{0}$ is a pre-exponential factor, $E_{A}$ the activation energy of the process, and $R$ the ideal gas constant. The Arrhenius fits are shown in Figure 5a by the different continuous lines, while the fitting parameters are summarized in Table 2 . The $\beta_{1}$ process showed in all cases a similar pre-exponential factor around $10^{-15} \mathrm{~s}$, and activation energies slightly depending on the thermal treatment. We observed that FC-PPeF presented the highest $E_{A}$ value, which decreased for the SL-PPeF sample. Finally, the semicrystalline Aged-PPeF polymer showed the smallest $E_{A}$ value $(46 \mathrm{~kJ} / \mathrm{mol})$. The $\beta_{2}$ relaxation, only observed in FC-PPeF, presented a much higher $E_{A}$ value compared to the faster component. In this case, the pre-exponential factor was too low as compared to the reciprocal of a vibrational frequency $\left(\sim 10^{-13} \mathrm{~s}\right)$.

Figure $5 \mathrm{~b}$ shows the relaxation strength as a function of the temperature. We observed that the $\beta_{2}$ component, only detected for FC-PPeF, decreases rapidly with increasing temperature, until it is no longer well-resolved above $270 \mathrm{~K}$. On the contrary, the strength of the $\beta_{1}$ component is essentially the same for FC-PPeF and SL-PPeF showing in both cases a slight increase with temperature. Finally, the Aged-PPeF sample presented markedly smaller relaxation strength but with a similar temperature dependence. 

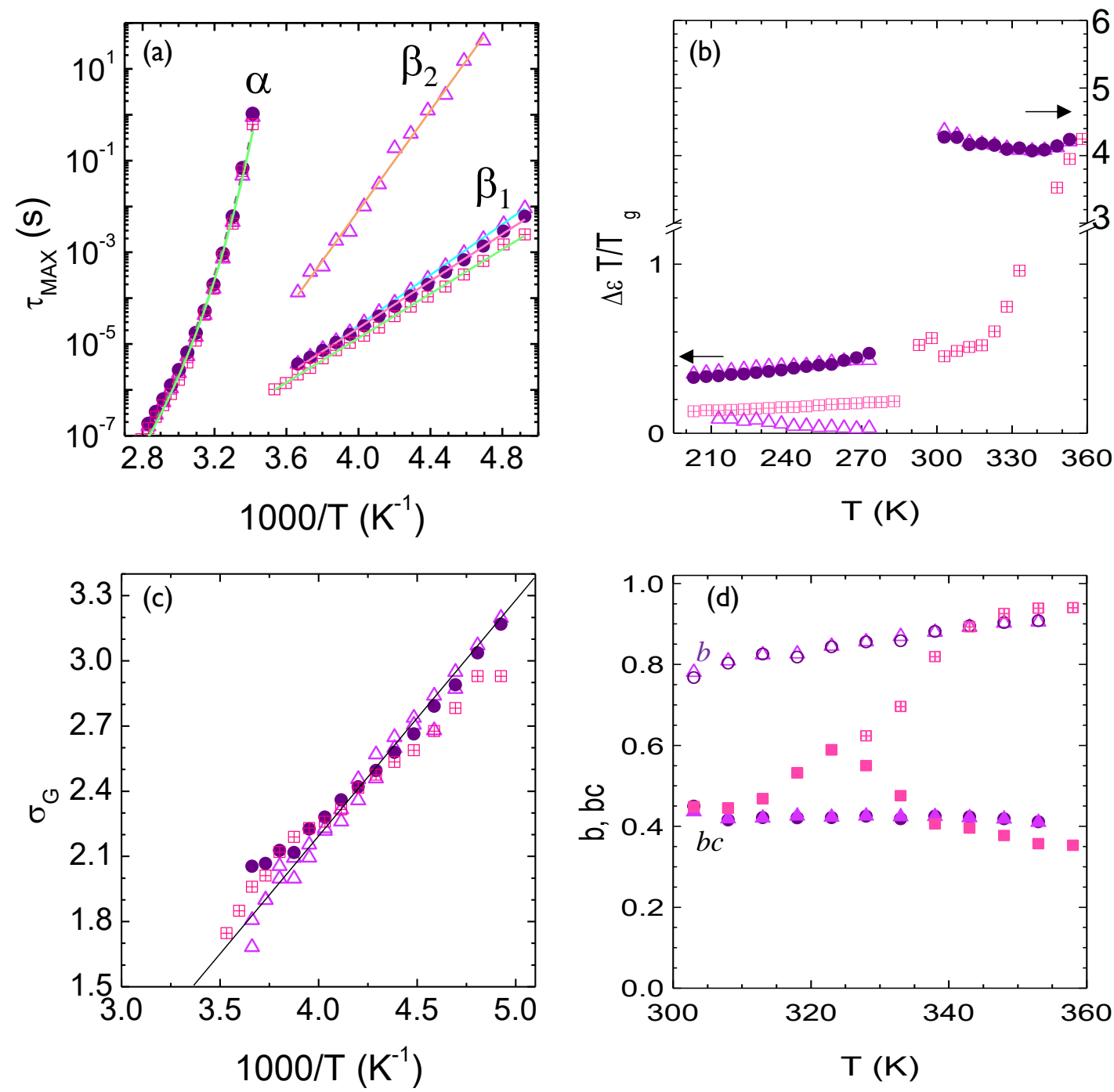

Figure 5. Temperature dependence of the fitting parameters used for modeling the BDS results: FC $(\triangle)$, SL (@), Aged (囚). (a) Relaxation plot, (b) relaxation intensity, (c) standard deviation of the Gaussian distribution of decimal logarithm of relaxation times for local relaxations, (d) shape parameters of the HN equation used to model the segmental relaxation ( $b$, open symbols; $b c$, filled symbols).

Table 2. Arrhenius and VFT parameters for all PPeF samples.

\begin{tabular}{|c|c|c|c|c|c|}
\hline \multirow{2}{*}{ Sample } & \multicolumn{2}{|c|}{$\beta$} & \multicolumn{3}{c|}{$\alpha$} \\
\cline { 2 - 3 } & $\begin{array}{c}\tau_{0} \\
(\mathrm{~s})\end{array}$ & $\begin{array}{c}E_{A} \\
(\mathrm{~kJ} / \mathrm{mol})\end{array}$ & $\begin{array}{c}\tau_{0-\mathrm{VFT}} \\
(\mathrm{s})\end{array}$ & $D$ & $\begin{array}{c}T_{\mathrm{VFT}} \\
(\mathrm{K})\end{array}$ \\
\hline FC-PPeF $\left(\beta_{1}\right)$ & $(2.2 \pm 0.4) \cdot 10^{-16}$ & $52 \pm 1$ & & & $251 \pm 1$ \\
\hline FC-PPeF $\left(\beta_{2}\right)$ & $(1.2 \pm 0.9) \cdot 10^{-24}$ & $104 \pm 2$ & \multirow{2}{*}{$10^{-12}$} & \multirow{2}{*}{5.1} & \\
\hline SL-PPeF & $(1.3 \pm 0.3) \cdot 10^{-15}$ & $49 \pm 1$ & & & $251 \pm 1$ \\
\hline Aged-PPeF & $(2.9 \pm 0.3) \cdot 10^{-15}$ & $46 \pm 1$ & & & $250.0 \pm 0.5$ \\
\hline
\end{tabular}


To analyze the shape of these local relaxations we calculated the standard deviation, $\sigma_{\mathrm{G}}$, of the distribution of Debye relaxations according to a Gaussian function of $\log _{10}(\tau)$, which corresponds to the symmetrical broadening of the $\mathrm{CC}$ functions fitting the data $(b$ in eqn $(1))$ :

$$
\sigma_{\mathrm{G}}=\frac{5 \cdot 10^{-4}}{b^{4}}-4 \cdot \log _{10}(b)
$$

A detailed description of eqn (4) is provided in the Supporting Information (Figure S4). The results obtained from this calculation are presented in Figure $5 \mathrm{c}$, as a function of inverse temperature. We observed that all the local relaxations presented the same trend, i.e., the relaxation peaks were narrower as temperature increased. The broadness of the local relaxations decreases linearly as a function of $1 / T$ from about 3.2 to 1.8 decades. Such a linear dependence is expected by assuming that the secondary relaxation is originated by superposition of single Debye processes with different time scales resulting from a Gaussian distribution of energy barriers separating equivalent local equilibrium states.

Segmental relaxation. At temperatures above $290 \mathrm{~K}$, the segmental $(\alpha)$ relaxation peak appeared in the BDS experimental frequency window. This is the main relaxation observed for all the samples in Figures $4 b-d$. The FC- and SL-PPeF samples presented intense peaks, with small but noticeable differences. Systematically, for all temperatures, the SL-PPeF showed a lower peak frequency. The Aged-PPeF sample presented the most different $\alpha$-relaxation. In this case, at $303 \mathrm{~K}$ and $333 \mathrm{~K}$, the relaxation peak showed a much lower intensity and a wider shape, while its maximum stayed at similar frequency, compared to the other two samples. At $348 \mathrm{~K}$, when $T>T_{\mathrm{m}}$, the relaxation peak intensity increased reaching the same value (also in shape and position) as the FC-PPeF sample. The segmental relaxation peaks were modeled using a HN 
function with a DC conductivity contribution, eqn (1), (see lines in Figure 4b-c). In the fitting procedure a $\mathrm{CC}$ function was used to account for the secondary relaxation contributions at high frequencies. The data, accompanied by the fitting components, are presented in Figure S3.

The temperature dependencies of the fitting parameters are shown in Figure 5. As already commented, there are small variations in the peak maxima between FC- and SLPPeF presented in Figure 5a. We described the relaxation times for all the samples using the Vogel-Fulcher-Tamman (VFT) law: ${ }^{57}$

$$
\tau_{\mathrm{MAX}}(T)=\tau_{0-\mathrm{VFT}} \exp \left[\frac{D T_{\mathrm{VFT}}}{T-T_{\mathrm{VFT}}}\right]
$$

where $\tau_{0-\mathrm{VFT}}$ is a pre-exponential factor, $D$ a parameter related to the so-called fragility (steepness around $T_{\mathrm{g}}$ ), and $T_{\mathrm{VFT}}$ the so-called Vogel temperature (usually $30-60 \mathrm{~K}$ below $T_{\mathrm{g}}$ ). We found first the $\tau_{0-\mathrm{VFT}}$ and $D$ values by fitting the data in a broad temperature and frequency range, as detailed in Ref ${ }^{61}$. The obtained $D$ value is comparable to that previously found for PPeF by Papamokos and collaborators ${ }^{23}$. The $T_{\mathrm{VFT}}$ values that resulted by maintaining $\tau_{0-\mathrm{VFT}}$ and $D$ fixed in the VFT equation are summarized in Table 2. $T_{\mathrm{VFT}}$ values were around $250 \mathrm{~K}$ varying in line with the calorimetric glass transition temperatures, and similar to that previously reported $\left(247 \mathrm{~K}, \operatorname{Ref}^{23}\right)$.

The $\alpha$ relaxation strength, shown in Figure 5b, was almost constant for both FCand SL-PPeF, being the latter slightly lower for all temperatures. The intensity of the Aged-PPeF segmental relaxation peak was much smaller but increased with temperature, as the sample enters in the melting range observed in DSC. Above the melting range, it remained constant and equal to that of FC-PPeF film. Finally, the shape parameters 
(Figure 5d) were similar for both the FC- and SL-PPeF samples, being weakly temperature dependent. The Aged-PPeF sample showed a non-monotonous marked temperature dependence, related to the transformation from the semicrystalline solid state at low temperatures to the melt.

\section{Discussion}

PPeF showed no signs of crystallization even after slow cooling from the melt, by both DSC and XRD, unlike the results presented for other furan-based polymers with a shorter glycolic subunit, as PEF, ${ }^{62-63} \mathrm{PTF}^{64}$ and $\mathrm{PBF},{ }^{58}$ as well as for some of them with longer glycolic chains. ${ }^{23}$ Nonetheless, we observed that bulk PPeF undergoes cold crystallization at room temperature with extremely slow kinetics, slower than those detectable in usual laboratory times. This result is in line with recent AFM observations of PPeF crystallization in thin film geometry, where crystalline structures started appearing after 3 months of storage at room temperature. ${ }^{55}$ To provide further information about the crystallization behavior of bulk PPeF, in our current work we performed DSC and XRD studies of a freshly prepared sample as a function of the storage time, as presented in Figure S1. There, it is observed that the PPeF film requires about 4 months to develop some detectable crystalline structures. This could be the reason why in previous reports this polymer has been considered as fully amorphous in the bulk. ${ }^{23-24,56}$ Crystallinity values $\left(X_{c}\right)$, calculated from XRD scans, were $3 \pm 1 \%, 7 \pm 2 \%$ and $17 \pm 2$ $\%$ for samples with storage times of 4,10 and 12 months, respectively. Concerning XRD results, we highlight that in the article by Guidotti and collaborators, the authors detected a sharp and intense reflection at $q=1.26 \AA^{-1}$ for an amorphous PPeF sample. ${ }^{24}$ This peak was observed to be independent of thermal history of the sample, and its nature was attributed to the formation of a mesophase. ${ }^{24}$ In the Aged-PPeF sample, we do not observe a reflection at this specific angle; however, there is a really close one located at $q=1.23$ 
$\AA^{-1}$ (black arrow in Figure $3 b$ ) that could mask it. Contrary to the previous work, this reflection disappears once the polymer sample is heated above the calorimetric $T_{\mathrm{m}}$ suggesting it is related to the crystal phase.

After cold crystallization, the amorphous phase of the PPeF is affected in different ways by the presence of surrounding crystals. DSC results showed a broader glass transition of Aged-PPeF in the same temperature range. The $\Delta c_{\mathrm{p}}$ also showed a decrease in comparison to that corresponding to the other thermal treatments. From this result, one can calculate the mobile amorphous fraction in the Aged-PPeF sample as $\chi_{a, m}=$ $\Delta c_{\mathrm{p}}^{\mathrm{Aged}-\mathrm{PPeF}} / \Delta c_{\mathrm{p}}^{\mathrm{FC}-\mathrm{PPeF}}=0.74$. This result would indicate that about $26 \%$ of the sample should be comprised by immobile phases, as crystals and rigid amorphous fractions. The crystallinity index obtained from $\mathrm{XRD}\left(\mathrm{X}_{\mathrm{c}}=17 \%\right)$ takes into account only the amount of material in crystal form and can be taken as a support for this result.

From the molecular dynamics point of view, PPeF showed clear changes in the local relaxations depending on thermal history. The FC-PPeF sample presented an asymmetric response more extended towards lower frequencies, which was modeled by the sum of two CC components. This result is in line with our previous BDS measurements on several melt-quenched poly(alkylene 2,5-furanoate)s. ${ }^{56,58}$ In these latter works, the faster component was related to the $\mathrm{O}-\mathrm{C}$ bond of the ester oxygen to the aliphatic carbon, while the slower one to the $\mathrm{C}-\mathrm{CA}$ link between the ester group carbon and the furan ring. The activation energies found for these two relaxations, in FC-PPeF, indicate that the two local motions used to describe the sub-glass relaxation should correspond to those previously described. Concerning the relaxation strength of these two components, we noticed that $\Delta \varepsilon_{\beta_{1}} \gg \Delta \varepsilon_{\beta_{2}}$, indicating that the whole $\beta$ relaxation was dominated by the faster component, a result also in line with previous reports. ${ }^{56,58}$ 
After slow cooling from the melt, a single $\mathrm{CC}$ component was sufficient to describe the $\beta$ relaxation of PPeF (SL-PPeF sample). This CC function related quite well to the $\beta_{1}$ relaxation component of the fast cooled sample, considering the obtained parameters (Figure 5). From the relaxation map, we observed that $E_{\mathrm{A}}$ only showed a small decrease, coinciding with the one reported by Papamokos et al., where a singlecomponent local relaxation was observed for PPeF. ${ }^{23}$ Also, Soccio et al. found a single local relaxation for several poly(alkylene 2,5-furanoate) samples, including PPeF, cooled at $3 \mathrm{~K} / \mathrm{min}$ from the melt. ${ }^{56}$ To evaluate the dependence of the local relaxation asymmetry with cooling rate, we subjected the PPeF to different thermal protocols. The corresponding results are presented in Figure 6.

First, Figure 6a shows the BDS results at $218 \mathrm{~K}\left(T \ll T_{\mathrm{g}}\right)$ for the FC- and SLsamples, as well as for two additional thermal protocols represented by solid squares and solid stars. These addition protocols correspond to PPeF samples cooled down from 453 to $153 \mathrm{~K}$, at $50 \mathrm{~K} / \mathrm{min}$ and $3 \mathrm{~K} / \mathrm{min}$, respectively, values that lie in between the rate of fast and slow cooling tests. In both these new cases, the BDS measurements were carried out on heating from $203 \mathrm{~K}$, in $5 \mathrm{~K}$ isothermal steps. We observed that the dielectric loss asymmetry, indicative of the intensity of the $\beta_{2}$ process, was reduced as cooling rate was reduced. Thus, as the system cools down from the melt, the blocking of the molecular motions related to the $\beta_{2}$ relaxation increased with the time that the system was allowed to equilibrate. From the molecular point of view, our present work, in combination with literature reports, ${ }^{56,58}$ show that in a well-equilibrated sample there is a blocking of the motion involving the $\mathrm{C}-\mathrm{CA}$ link.

Second, trying to have a better understanding of the origin of this unusual phenomenon, we performed the following experiment. The PPeF sample was heated to $453 \mathrm{~K}$ and melt-quenched into liquid $\mathrm{N}_{2}$, as for the FC-PPeF sample discussed so far. 
Then, the sample was measured in $5 \mathrm{~K}$ isothermal steps up to $218 \mathrm{~K}$. The data collected at this temperature are depicted in Figure $6 b$ by the pink circles with the highest intensity. Then, the BDS experiments proceed as follows. The sample was heated to $223 \mathrm{~K}$, a new BDS measurement was performed isothermally, as usual, and then the sample was cooled down to $218 \mathrm{~K}$ to be re-measured. This protocol was repeated by increasing the so-called "temperature of treatment" ( $\left.T_{\text {treatment }}\right) 5 \mathrm{~K}$ higher each time, and always cooling down to $218 \mathrm{~K}$ to check the response evolution. A schematic representation of the protocol is presented in Figure S5. We observed a monotonic decrease of the low frequency losses, at $218 \mathrm{~K}$, as $T_{\text {treatment }}$ increased. In the inset of Figure $6 \mathrm{~b}$, we show the maximum of the

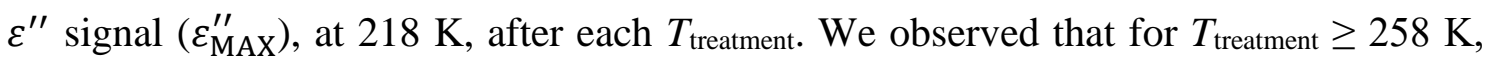
$\varepsilon_{\text {MAX }}^{\prime \prime}$ reached a constant value, somehow indicating that the system did not further evolve. These results seem to be directly connected to the continuous decreasing of the relaxation strength of the $\beta_{2}$ component as temperature increases and the fact that it is no longer detectable at $T_{\text {treatment }} \geq 270 \mathrm{~K}$.
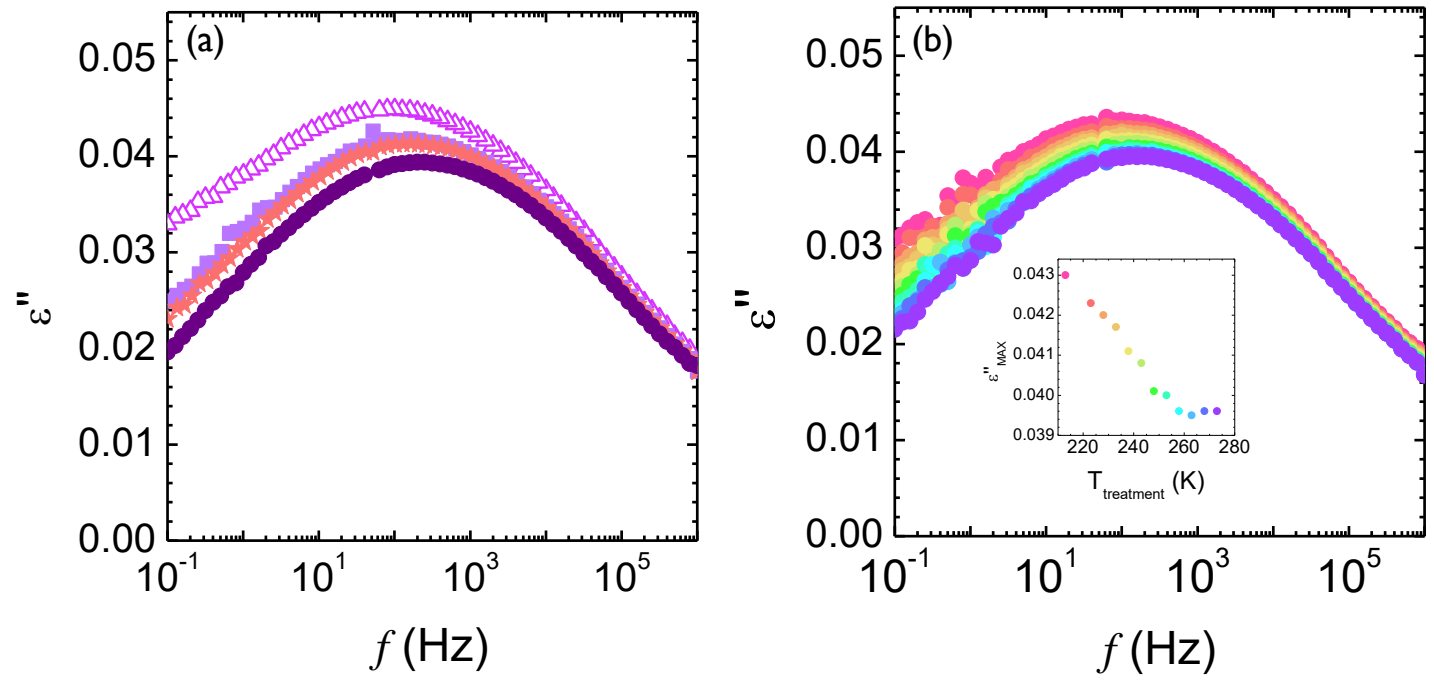

Figure 6. Dielectric losses at $218 \mathrm{~K}$, (a) after cooling from $423 \mathrm{~K}$ at different rates: FC-PPeF ( $\triangle$ ), $50 \mathrm{~K} / \mathrm{min}$ $(\boldsymbol{\square}), 3 \mathrm{~K} / \mathrm{min}(\star)$, SL-PPeF (•), and (b) after progressive annealing. Inset in panel (b) shows the evolution of $\varepsilon_{\mathrm{MAX}}^{\prime \prime}$ as a function of treatment temperature. 
Considering that the low frequency loss component of the local relaxation arises from a rather specific molecular motion, it becomes really interesting that its evolution takes place below $T_{\mathrm{g}}$ where the polymer is in a low-mobility state. It is worth noticing that this "local equilibration", occurring well below the glass-transition range, does not result in significant differences in the DSC signals of FC- and SL- samples, both showing approximately the same glass transition temperature and $\Delta c_{\mathrm{p}}$ values. Concerning the segmental relaxation, we only detected tiny differences, as illustrated in Figure 4. Interestingly, the SL-PPeF sample presents a small excess of losses in the low frequency flank of the $\alpha$-relaxation loss peak, with respect to that of the FC sample. As a result, the peak position appears shifted towards lower frequencies and the full-width-at-halfmaximum of the loss peak slightly increases. These effects persist until a rather high temperature, $343 \mathrm{~K}$, which is well above $T_{\mathrm{g}}$. This result would be indicative of the occurrence of some structural development in the SL-PPeF, which is prevented (at least in part) by fast cooling.

In order to detect the presence of any structural feature in PPeF, we have performed linear rheological experiments by means of dynamic torsional shear tests in the temperature range 313 - $393 \mathrm{~K}$, i.e. above its $T_{\mathrm{g}}$. The results, depicted in Figure 7, show that the time-temperature superposition (TTS) $)^{65-67}$ can be reasonably applied to obtain a master curve, with shift factors following the expected Williams-Landel-Ferry (WLF) law $^{67}$ (see line in the inset). From this master curve, it is inferred that the PPeF melt does not behave as a conventional linear polymer, since it is found that Newtonian flow cannot be attained even at temperatures higher than $T_{\mathrm{g}}+100 \mathrm{~K}$. Instead, both $\mathrm{G}^{\prime}$ and G" present a parallel behavior at low frequencies approaching to a power law with exponent $2 / 3$. This finding reminds that of randomly branched polymers, ${ }^{68}$ for which at the gel point $G^{\prime} \sim G^{\prime \prime} \sim \omega^{2 / 3}$. This result could be related to previous ones showing that 
amorphous PPeF presents non-conventional mechanical and gas transport properties at room temperature. ${ }^{24}$

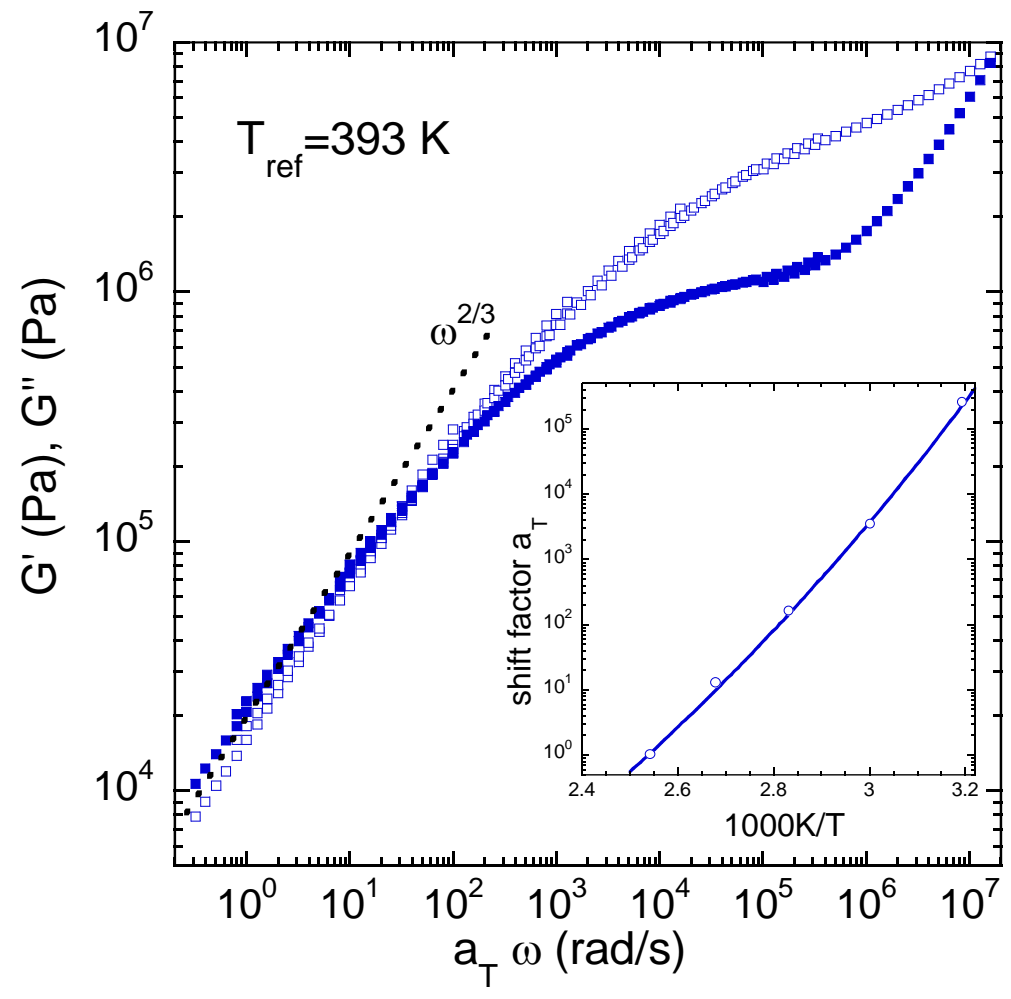

Figure 7. Master curve obtained from the dynamic experiments on PPeF using (linear) torsional shear: G' empty symbols, G" solid symbols. The inset presents the horizontal shift factors used and the line shows the WLF equation fitting the data.

Given the linear nature of the synthetized polymer, the obtained results suggest the presence of some kind of supramolecular structures in the PPeF molten state. Literature results on PEF have pointed out the presence of specific intermolecular interactions between neighboring furan moieties, as hydrogen-bonding and/or $\pi-\pi$ stacking. ${ }^{23-25,69}$ If this type of interactions exists in the PPeF melt, as recently proposed, ${ }^{24}$ they could give rise to a network structure, which would explain the observed anomalous behaviors and particularly the PPeF rheology. 
In order to detect any additional evidence of such specific intermolecular interactions in the amorphous PPeF melt, we have performed FTIR experiments, at 230 $\mathrm{K}$, on PPeF films to compare the absorption spectrum of FC- and SL-PPeF samples. We found that there are minor but significant and reproducible differences. As presented in Figure 8, FTIR shows that a weak additional absorption at $670 \mathrm{~cm}^{-1}$ appears in the SLPPeF only, together with some excess of absorption in the ranges $700-750 \mathrm{~cm}^{-1}, 1500$ $1550 \mathrm{~cm}^{-1}$ and $3000-3450 \mathrm{~cm}^{-1}$. Note that the absorption at these higher frequencies is mainly originated by the $\mathrm{C}-\mathrm{H}$ stretching of the furan rings that are assumed to be involved in the specific interactions. All these results support the idea that there exist some specific interlinkages that would give rise to local supramolecular structures in PPeF molten state and that the development of such specific interactions depends on the way the polymer is cooled from high temperatures.

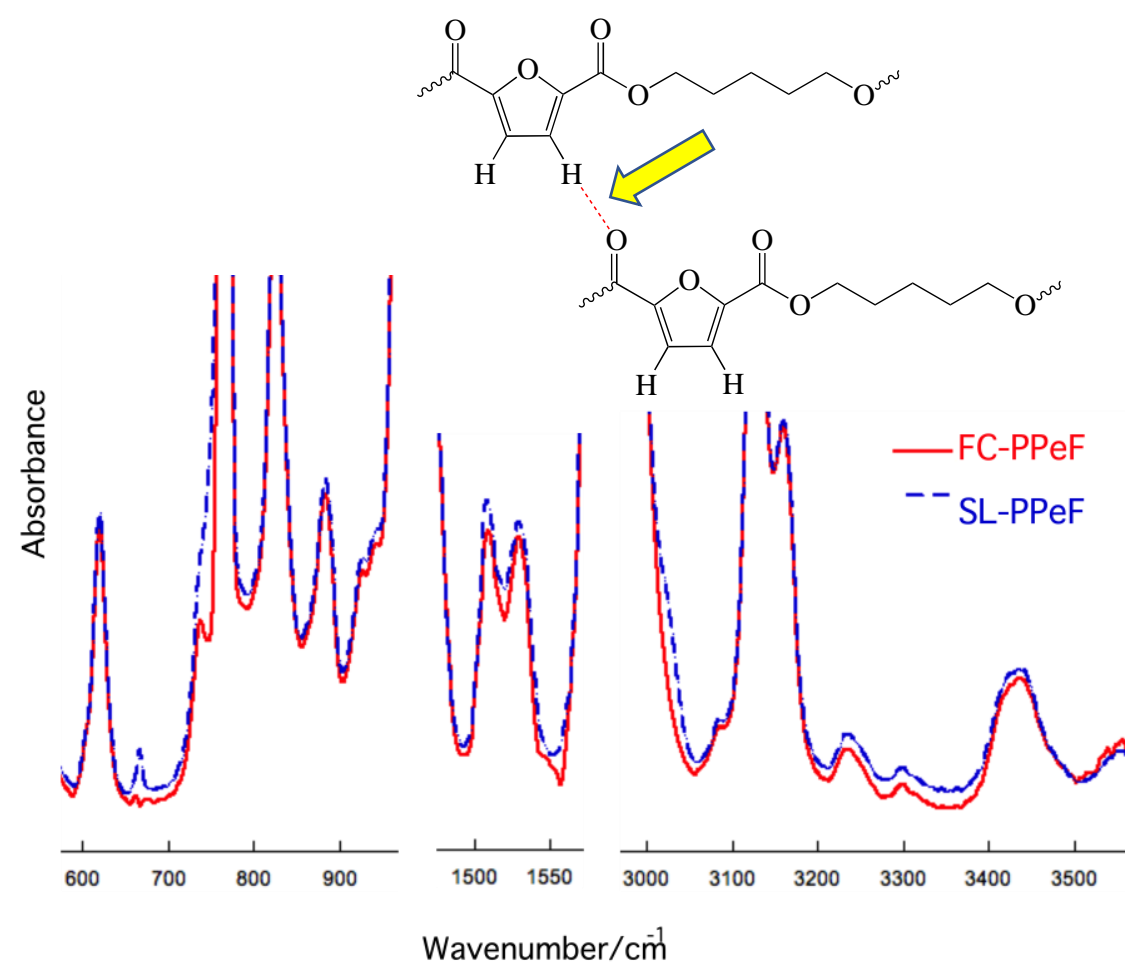

Figure 8. Effect of the cooling protocol on the room temperature infrared absorption of a PPeF film. The three selected ranges where significant differences are observed are presented. The scheme presented as inset illustrates one of the possible interactions responsible for such FTIR differences, as suggested in Ref 24 
The presence of local supramolecular structures by slow cooling PPeF, as suggested by BDS, Rheology and FTIR measurements, would impact to some extent the segmental dynamics. According to our findings, the slower component of the $\alpha$ relaxation times is enhanced, which could be explained by some induced correlation in dipole orientation between neighboring entities around the sites where the specific interactions are stronger. The same picture could explain the behavior observed in the secondary relaxation (see Figure 6). By fast cooling PPeF, the development of specific interactions would be partially prevented. As evidenced by the results of the experiment summarized in Figure S5, by heating the FC-PPeF sample to $243 \mathrm{~K}$, the local molecular mobility could be sufficient to allow some conformational changes favoring specific interactions, which would result in a reduction of the slower secondary relaxation component.

The above arguments could also explain the fact that the reduction in the $\beta$ relaxation intensity observed in the semicrystalline polymer (Aged-PPeF) is far larger than that expected. Particularly, as thoughtfully discussed in previous publications, ${ }^{58,}$, 72 polymer crystallinity affects the local relaxation, mostly in their relaxation strength without other significant changes, usually. In this way, one could expect the following relation:

$$
\chi_{\mathrm{c}} \approx 1-\frac{\varepsilon_{\beta-\text { Semicrys }}^{\prime \prime}}{\varepsilon_{\beta-\text { Amorph }}^{\prime \prime}}
$$

where $\chi_{\mathrm{c}}$ would be a measure of the crystallinity, and $\varepsilon_{\beta \text {-Semicrys }}^{\prime \prime}$ and $\varepsilon_{\beta}^{\prime \prime}$-Amorph the local relaxations for the semicrystalline and amorphous samples, respectively. However, the results from Aged-PPeF showed a quite smaller local relaxation as compared to SL-PPeF. For instance, at $243 \mathrm{~K}$, we found: $1-\frac{\varepsilon_{\beta-\text { Semicrys }}^{\prime \prime}}{\varepsilon_{\beta}^{\prime \prime} \text {-Amorph }} \approx 0.58$ a value much higher than that estimated by XRD (17\%). 
This much larger reduction of the secondary relaxation strength could be reflecting the structural features discussed above that would modify local packing prior crystal formation. However, in the case of Aged-PPeF this effect can also be due to partial chain orientation occurring during the film preparation. ${ }^{24}$ In order to test the genuine effect of crystallinity on the local relaxation, we performed new BDS measurements on an already measured PPeF sample that was subsequently maintained between the two electrodes for 2 years. The sample capacitor was left in a desiccator, at room temperature, and previously the new BDS experiments it was subjected to high vacuum for 48 hours to remove any possible trace of moisture. The new BDS measurements were performed starting from $183 \mathrm{~K}$ up to $373 \mathrm{~K}$, in $5 \mathrm{~K}$ isothermal steps. Then, the sample was cooled in $10 \mathrm{~K}$ isothermal steps, down to $183 \mathrm{~K}$, and re-measured on heating again up to $373 \mathrm{~K}$. We also performed corresponding DSC measurements by collecting c.a. $5 \mathrm{mg}$ of the polymer that was surrounding the smaller electrode, prior the new BDS experiments. The obtained results are presented in Figure 9, together with the calorimetric traces of the PPeF fast cooled-down from the melt.
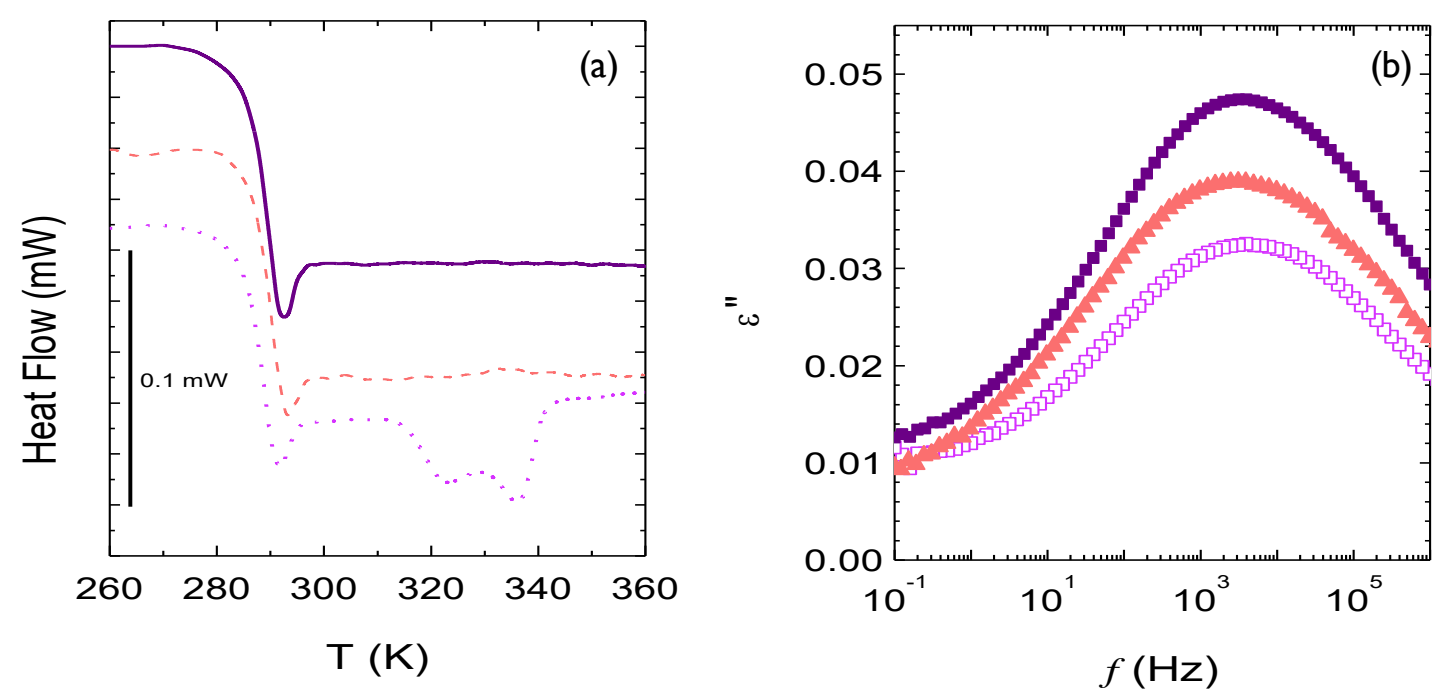

Figure 9. (a) DSC results (exo up) of PPeF: aged for 2 years at room temperature inside a BDS capacitor (dotted line), fast cooled from the melt (continuous line) and aged for 3 months at room temperature (dashed line). (b) Dielectric loss spectra, at $243 \mathrm{~K}$, of the PPeF aged for 2 years at room temperature in a BDS 
capacitor (empty squares), fast cooled from $370 \mathrm{~K}$ after melting (solid squares) and aged for 3 months at room temperature (solid triangles).

The DSC results showed the glass transition temperature at around $289 \mathrm{~K}$ for both heating runs, and the $\Delta c_{\mathrm{p}}$ values were the same $(0.37 \mathrm{~J} / \mathrm{g} \cdot \mathrm{K})$ within error. Considering the endothermic melting peaks above $310 \mathrm{~K}$, we calculated a melting enthalpy of $3 \mathrm{~J} / \mathrm{g}$, much lower than the one observed for the Aged-PPeF sample $(23 \mathrm{~J} / \mathrm{g})$. This new value would correspond to a $2 \%$ crystallinity, extrapolating from DSC and XRD results. However, comparing BDS results from the local relaxations we obtained $1-\frac{\varepsilon_{\beta-\text { Semicrys }}^{\prime \prime}}{\varepsilon_{\beta \text {-Amorph }}^{\prime \prime}} \approx 0.33$. This clearly probes that most of the reduction in the PPeF local-relaxation intensity cannot be accounted by the freezing of local molecular motions in the crystalline phase, pointing to the relevance of the specific interactions affecting local packing.

To further corroborate this result, we performed additional BDS measurements on the same PPeF sample, which was subsequently maintained between the two electrodes for 3 months after melting. The BDS measurements were performed using the same protocol used before and were accompanied by corresponding DSC measurements. The obtained results are also presented in Figure 9. As can be seen, after 3 months the DSC trace showed no signs of crystallization, but the local relaxation presented an evident reduction of intensity, about half of that found after 2 years of aging. Furthermore, this clear change in the local dielectric relaxation was accomplished by only a very minor variation of the main dielectric relaxation intensity in the vicinity of $T_{\mathrm{g}}$, comparable to the differences observed above between the fast and slow cooled samples (Figure S6). Therefore, these results evidence that there are specific changes in PPeF occurring both below and above $T_{\mathrm{g}}$; particularly, the changes occurring at room temperature over a period 
of a few months affect significantly the local dielectric relaxation intensity prior to the development of any detectable crystalline phase.

\section{Conclusions}

PPeF has a very peculiar behavior compared to the other members of the homologous family, showing no sign of crystallization in regular time scales, but developing a measurable crystallinity when stored at room temperature for months or years depending on sample preparation methods. The crystalline phase so obtained melts at about $333 \mathrm{~K}$, i.e. at a temperature much lower than those observed for other furancontaining polyesters, which is indicative of a defective character of the crystals, despite the significant crystallinity. BDS analysis has shown that PPeF molecular dynamics, both at local and segmental range, are sensitive to how fast the material is cooled from high temperatures. The evidenced behavior has been attributed to the development of illdefined structures in the melt, likely related with the presence of specific intermolecular interactions involving the furan rings. Evidences of such local structures have been observed by tiny but reproducible changes in the infrared absorption spectrum. Intermolecular interactions could also be involved in the particular rheological behavior of PPeF, showing a gel-like behavior at temperatures as high as $T_{\mathrm{g}}+100 \mathrm{~K}$. Moreover, structural changes occur in PPeF during room temperature storage in months' scale, which reduce significantly the local molecular mobility prior the development of any crystalline structure.

\section{Acknowledgements}

A. A. acknowledges funding from Spanish Government "Ministerio de Ciencia, Innovacion y Universidades" (PGC2018-094548-B-I00 (MCIU/ AEI/FEDER, UE)). A. A. and B. R. acknowledge funding by Basque Government (IT-1175-19). D.E.M. acknowledges the financial support obtained by the MCIU fellowship "Juan de la Cierva - Incorporación" (IJCI- 
2017-31600). This research was funded by the European Union: EUSMI, H2020-INFRAIA2016-1, PROJECT 731019, via proposals E171100043 and E171100040. Authors acknowledge Dr. Elodie Limousin assistance for preparation of PPeF cross-sections. The authors thank Dr. Amaia Iturrospe for assistance in X-ray scattering measurements and Dr. Silvia Arrese-Igor for assistance in FTIR measurements. This publication is based upon work from Cost Action FUR4Sustain, supported by COST.

\section{References}

1. Plastics: the Facts - An analysis of European plastics production, demand and waste data. . www.plasticseurope.org/.

2. Geyer, R.; Jambeck, J. R.; Law, K. L., Production, use, and fate of all plastics ever made. Science Advances 2017, 3 (7), e1700782.

3. Bioplastics packaging: combining performance with sustainability - Materials and market development in the packaging segment. www.european-bioplastics.org.

4. Directorate-General for Environment (European Commission). Being wise with waste: The EU's approach to waste management. Publications Office of the EU: 2010.

5. Directive 2008/98/EC on waste (Waste Framework Directive). 2008.

6. Al-Salem, S. M.; Lettieri, P.; Baeyens, J., Recycling and recovery routes of plastic solid waste (PSW): A review. Waste Management 2009, 29 (10), 2625-2643.

7. Siracusa, V.; Rocculi, P.; Romani, S.; Rosa, M. D., Biodegradable polymers for food packaging: a review. Trends in Food Science \& Technology 2008, 19 (12), 634-643.

8. Hedenqvist, M. S., Chapter 26 - Barrier packaging materials. In Handbook of Environmental Degradation of Materials, Kutz, M., Ed. William Andrew Publishing: Norwich, NY, 2005; pp 547-563.

9. Emblem, A., 13 - Plastics properties for packaging materials. In Packaging Technology, Emblem, A.; Emblem, H., Eds. Woodhead Publishing: 2012; pp 287-309.

10. Robertson, G. L., Optical, Mechanical and Barrier Properties of Thermoplastics Polymers. In Food Packaging-Principles and Practice, Taylor \& Francis: 2013; pp 91-130.

11. Wagner, J. R.; Marks, S. B., Multilayer Flexible Packaging. Elseviers: 2016.

12. Nakajima, H.; Dijkstra, P.; Loos, K., The Recent Developments in Biobased Polymers toward General and Engineering Applications: Polymers that are Upgraded from Biodegradable Polymers, Analogous to Petroleum-Derived Polymers, and Newly Developed. Polymers 2017, 9 (10), 523.

13. Rabnawaz, M.; Wyman, I.; Auras, R.; Cheng, S., A roadmap towards green packaging: the current status and future outlook for polyesters in the packaging industry. Green Chemistry 2017, 19 (20), 4737-4753.

14. Jiang, Y.; Woortman, A. J. J.; Alberda van Ekenstein, G. O. R.; Loos, K., A biocatalytic approach towards sustainable furanic-aliphatic polyesters. Polymer Chemistry 2015, 6 (29), 5198-5211.

15. Sousa, A. F.; Vilela, C.; Fonseca, A. C.; Matos, M.; Freire, C. S. R.; Gruter, G.-J. M.; Coelho, J. F. J.; Silvestre, A. J. D., Biobased polyesters and other polymers from 2,5-furandicarboxylic acid: a tribute to furan excellency. Polymer Chemistry 2015, 6 (33), 5961-5983.

16. Morales-Huerta, J. C.; Martínez de Ilarduya, A.; Muñoz-Guerra, S., Poly(alkylene 2,5furandicarboxylate)s (PEF and PBF) by ring opening polymerization. Polymer 2016, 87, 148-158. 
17. Papageorgiou, G. Z.; Papageorgiou, D. G.; Terzopoulou, Z.; Bikiaris, D. N., Production of bio-based 2,5-furan dicarboxylate polyesters: Recent progress and critical aspects in their synthesis and thermal properties. European Polymer Journal 2016, 83, 202-229.

18. Terzopoulou, Z.; Tsanaktsis, V.; Nerantzaki, M.; Papageorgiou, G. Z.; Bikiaris, D. N., Decomposition mechanism of polyesters based on 2,5-furandicarboxylic acid and aliphatic diols with medium and long chain methylene groups. Polymer Degradation and Stability 2016, 132, 127-136.

19. Papageorgiou, D. G.; Guigo, N.; Tsanaktsis, V.; Exarhopoulos, S.; Bikiaris, D. N.; Sbirrazzuoli, N.; Papageorgiou, G. Z., Fast Crystallization and Melting Behavior of a Long-Spaced Aliphatic Furandicarboxylate Biobased Polyester, Poly(dodecylene 2,5-furanoate). Industrial \& Engineering Chemistry Research 2016, 55 (18), 5315-5326.

20. Tsanaktsis, V.; Terzopoulou, Z.; Nerantzaki, M.; Papageorgiou, G. Z.; Bikiaris, D. N., New poly(pentylene furanoate) and poly(heptylene furanoate) sustainable polyesters from diols with odd methylene groups. Materials Letters 2016, 178, 64-67.

21. Genovese, L.; Lotti, N.; Siracusa, V.; Munari, A., Poly(Neopentyl Glycol Furanoate): A Member of the Furan-Based Polyester Family with Smart Barrier Performances for Sustainable Food Packaging Applications. Materials 2017, 10 (9), 1028.

22. Guidotti, G.; Soccio, M.; Lotti, N.; Gazzano, M.; Siracusa, V.; Munari, A., Poly(propylene 2,5-thiophenedicarboxylate) vs. Poly(propylene 2,5-furandicarboxylate): Two Examples of High Gas Barrier Bio-Based Polyesters. Polymers 2018, 10 (7), 785.

23. Papamokos, G.; Dimitriadis, T.; Bikiaris, D. N.; Papageorgiou, G. Z.; Floudas, G., Chain Conformation, Molecular Dynamics, and Thermal Properties of Poly(n-methylene 2,5furanoates) as a Function of Methylene Unit Sequence Length. Macromolecules 2019, 52 (17), 6533-6546.

24. Guidotti, G.; Soccio, M.; García-Gutiérrez, M.-C.; Gutiérrez-Fernández, E.; Ezquerra, T. A.; Siracusa, V.; Munari, A.; Lotti, N., Evidence of a 2D-Ordered Structure in Biobased Poly(pentamethylene furanoate) Responsible for Its Outstanding Barrier and Mechanical Properties. ACS Sustainable Chemistry \& Engineering 2019, 7 (21), 17863-17871.

25. Guidotti, G.; Soccio, M.; García-Gutiérrez, M. C.; Ezquerra, T.; Siracusa, V.; GutiérrezFernández, E.; Munari, A.; Lotti, N., Fully Biobased Superpolymers of 2,5-Furandicarboxylic Acid with Different Functional Properties: From Rigid to Flexible, High Performant Packaging Materials. ACS Sustainable Chemistry \& Engineering 2020, 8 (25), 9558-9568.

26. Werpy, T.; Petersen, G., Top Value Added Chemicals from Biomass: Volume I -- Results of Screening for Potential Candidates from Sugars and Synthesis Gas. (US), U. D. o. E., Ed. 2004. 27. Boisen, A.; Christensen, T. B.; Fu, W.; Gorbanev, Y. Y.; Hansen, T. S.; Jensen, J. S.; Klitgaard, S. K.; Pedersen, S.; Riisager, A.; Ståhlberg, T.; Woodley, J. M., Process integration for the conversion of glucose to 2,5-furandicarboxylic acid. Chemical Engineering Research and Design 2009, 87 (9), 1318-1327.

28. de Jong, E.; Dam, M. A.; Sipos, L.; Gruter, G. J. M., Furandicarboxylic Acid (FDCA), A Versatile Building Block for a Very Interesting Class of Polyesters. In Biobased Monomers, Polymers, and Materials, American Chemical Society: 2012; Vol. 1105, pp 1-13.

29. van Putten, R.-J.; van der Waal, J. C.; de Jong, E.; Rasrendra, C. B.; Heeres, H. J.; de Vries, J. G., Hydroxymethylfurfural, A Versatile Platform Chemical Made from Renewable Resources. Chemical Reviews 2013, 113 (3), 1499-1597.

30. Ray, P.; Smith, C.; Simon, G. P.; Saito, K., Renewable Green Platform Chemicals for Polymers. Molecules 2017, 22 (3), 376.

31. Kohli, K.; Prajapati, R.; Sharma, B. K., Bio-Based Chemicals from Renewable Biomass for Integrated Biorefineries. Energies 2019, 12 (2), 233.

32. Zhang, J.; Liang, Q.; Xie, W.; Peng, L.; He, L.; He, Z.; Chowdhury, S. P.; Christensen, R.; Ni, Y., An Eco-Friendly Method to Get a Bio-Based Dicarboxylic Acid Monomer 2,5-Furandicarboxylic Acid and Its Application in the Synthesis of Poly(hexylene 2,5-furandicarboxylate) (PHF). Polymers 2019, 11 (2), 197. 
33. Knoop, R. J. I.; Vogelzang, W.; van Haveren, J.; van Es, D. S., High molecular weight poly(ethylene-2,5-furanoate); critical aspects in synthesis and mechanical property determination. Journal of Polymer Science Part A: Polymer Chemistry 2013, 51 (19), 4191-4199. 34. Hu, H.; Zhang, R.; Wang, J.; Ying, W. B.; Zhu, J., Synthesis and Structure-Property Relationship of Biobased Biodegradable Poly(butylene carbonate-co-furandicarboxylate). ACS Sustainable Chemistry \& Engineering 2018, 6 (6), 7488-7498.

35. Long, Y.; Zhang, R.; Huang, J.; Wang, J.; Jiang, Y.; Hu, G.-h.; Yang, J.; Zhu, J., Tensile Property Balanced and Gas Barrier Improved Poly(lactic acid) by Blending with Biobased Poly(butylene 2,5-furan dicarboxylate). ACS Sustainable Chemistry \& Engineering 2017, 5 (10), 9244-9253.

36. Hong, S.; Min, K.-D.; Nam, B.-U.; Park, O. O., High molecular weight bio furan-based copolyesters for food packaging applications: synthesis, characterization and solid-state polymerization. Green Chemistry 2016, 18 (19), 5142-5150.

37. Jia, Z.; Wang, J.; Sun, L.; Liu, F.; Zhu, J.; Liu, X., Copolyesters developed from bio-based 2,5-furandicarboxylic acid: Synthesis, sequence distribution, mechanical, and barrier properties of poly(propylene-co-1,4-cyclohexanedimethylene 2,5-furandicarboxylate)s. Journal of Applied Polymer Science 2019, 136 (13), 47291.

38. Xie, H.; Wu, L.; Li, B.-G.; Dubois, P., Modification of Poly(ethylene 2,5furandicarboxylate) with Biobased 1,5-Pentanediol: Significantly Toughened Copolyesters Retaining High Tensile Strength and O2 Barrier Property. Biomacromolecules 2019, 20 (1), 353364.

39. Guidotti, G.; Genovese, L.; Soccio, M.; Gigli, M.; Munari, A.; Siracusa, V.; Lotti, N., Block Copolyesters Containing 2,5-Furan and trans-1,4-Cyclohexane Subunits with Outstanding Gas Barrier Properties. International Journal of Molecular Sciences 2019, 20 (9), 2187.

40. Wu, L.; Mincheva, R.; Xu, Y.; Raquez, J.-M.; Dubois, P., High Molecular Weight Poly(butylene succinate-co-butylene furandicarboxylate) Copolyesters: From Catalyzed Polycondensation Reaction to Thermomechanical Properties. Biomacromolecules 2012, 13 (9), 2973-2981.

41. Zhou, W.; Wang, X.; Yang, B.; Xu, Y.; Zhang, W.; Zhang, Y.; Ji, J., Synthesis, physical properties and enzymatic degradation of bio-based poly(butylene adipate-co-butylene furandicarboxylate) copolyesters. Polymer Degradation and Stability 2013, 98 (11), 2177-2183.

42. Jacquel, N.; Saint-Loup, R.; Pascault, J.-P.; Rousseau, A.; Fenouillot, F., Bio-based alternatives in the synthesis of aliphatic-aromatic polyesters dedicated to biodegradable film applications. Polymer 2015, 59, 234-242.

43. Zhou, W.; Zhang, Y.; Xu, Y.; Wang, P.; Gao, L.; Zhang, W.; Ji, J., Synthesis and characterization of bio-based poly(butylene furandicarboxylate)-b-poly(tetramethylene glycol) copolymers. Polymer Degradation and Stability 2014, 109, 21-26.

44. Wu, B.; Xu, Y.; Bu, Z.; Wu, L.; Li, B.-G.; Dubois, P., Biobased poly(butylene 2,5furandicarboxylate) and poly(butylene adipate-co-butylene 2,5-furandicarboxylate)s: From synthesis using highly purified 2,5-furandicarboxylic acid to thermo-mechanical properties. Polymer 2014, 55 (16), 3648-3655.

45. Wang, J.; Liu, X.; Zhang, Y.; Liu, F.; Zhu, J., Modification of poly(ethylene 2,5furandicarboxylate) with 1,4-cyclohexanedimethylene: Influence of composition on mechanical and barrier properties. Polymer 2016, 103, 1-8.

46. Matos, M.; Sousa, A. F.; Silvestre, A. J. D., Improving the Thermal Properties of Poly(2,5furandicarboxylate)s Using Cyclohexylene Moieties: A Comparative Study. Macromolecular Chemistry and Physics 2017, 218 (5), 1600492.

47. Hu, H.; Zhang, R.; Sousa, A.; Long, Y.; Ying, W. B.; Wang, J.; Zhu, J., Bio-based poly(butylene 2,5-furandicarboxylate)-b-poly(ethylene glycol) copolymers with adjustable degradation rate and mechanical properties: Synthesis and characterization. European Polymer Journal 2018, 106, 42-52. 
48. Wang, J.; Liu, X.; Jia, Z.; Sun, L.; Zhu, J., Highly crystalline polyesters synthesized from furandicarboxylic acid (FDCA): Potential bio-based engineering plastic. European Polymer Journal 2018, 109, 379-390.

49. Wang, J.; Liu, X.; Jia, Z.; Liu, Y.; Sun, L.; Zhu, J., Synthesis of bio-based poly(ethylene 2,5furandicarboxylate) copolyesters: Higher glass transition temperature, better transparency, and good barrier properties. Journal of Polymer Science Part A: Polymer Chemistry 2017, 55 (19), 3298-3307.

50. Hu, H.; Zhang, R.; Shi, L.; Ying, W. B.; Wang, J.; Zhu, J., Modification of Poly(butylene 2,5furandicarboxylate) with Lactic Acid for Biodegradable Copolyesters with Good Mechanical and Barrier Properties. Industrial \& Engineering Chemistry Research 2018, 57 (32), 11020-11030.

51. Hu, H.; Zhang, R.; Wang, J.; Ying, W. B.; Zhu, J., Fully bio-based poly(propylene succinateco-propylene furandicarboxylate) copolyesters with proper mechanical, degradation and barrier properties for green packaging applications. European Polymer Journal 2018, 102, 101-110.

52. Guidotti, G.; Soccio, M.; Lotti, N.; Siracusa, V.; Gazzano, M.; Munari, A., New multi-block copolyester of 2,5-furandicarboxylic acid containing PEG-like sequences to form flexible and degradable films for sustainable packaging. Polymer Degradation and Stability 2019, 169, 108963.

53. Burgess, S. K.; Karvan, O.; Johnson, J. R.; Kriegel, R. M.; Koros, W. J., Oxygen sorption and transport in amorphous poly(ethylene furanoate). Polymer 2014, 55 (18), 4748-4756.

54. Burgess, S. K.; Kriegel, R. M.; Koros, W. J., Carbon Dioxide Sorption and Transport in Amorphous Poly(ethylene furanoate). Macromolecules 2015, 48 (7), 2184-2193.

55. Robles-Hernández, B.; Soccio, M.; Castrillo, I.; Guidotti, G.; Lotti, N.; Alegría, A.; Martínez-Tong, D. E., Poly(alkylene 2,5-Furanoate)s Thin Films: Morphology, Crystallinity and Nanomechanical Properties. Polymer 2020.

56. Soccio, M.; Martínez-Tong, D. E.; Guidotti, G.; Robles-Hernández, B.; Munari, A.; Lotti, N.; Alegria, A., Broadband Dielectric Spectroscopy Study of Biobased Poly(alkylene 2,5furanoate)s' Molecular Dynamics. Polymers 2020, 12 (6), 1355.

57. Kremer, F.; Schönhals, A., Broadband Dielectric Spectroscopy. 1 ed.; Springer-Verlag Berlin Heidelberg: 2003; $\mathrm{p} \mathrm{XXI,} 729$.

58. Soccio, M.; Martínez-Tong, D. E.; Alegría, A.; Munari, A.; Lotti, N., Molecular dynamics of fully biobased poly(butylene 2,5-furanoate) as revealed by broadband dielectric spectroscopy. Polymer 2017, 128, 24-30.

59. Cui, J.; Nogales, A.; Ezquerra, T. A.; Rebollar, E., Influence of substrate and film thickness on polymer LIPSS formation. Applied Surface Science 2017, 394, 125-131.

60. Rodríguez-Beltrán, R. I.; Martínez-Tong, D. E.; Reyes-Contreras, A.; Paszkiewicz, S.; Szymczyk, A.; Ezquerra, T. A.; Moreno, P.; Rebollar, E., Laterally-resolved mechanical and tribological properties of laser-structured polymer nanocomposites. Polymer 2019, 168, 178184.

61. Soccio, M.; Martínez-Tong, D. E.; Robles-Hernández, B.; Guidotti, G.; Munari, A.; Lotti, N.; Alegría, A., Broadband dielectric spectroscopy for the study of bio-based poly(alkylene furanoate)s' molecular dynamics. In preparation 2020.

62. Dimitriadis, T.; Bikiaris, D. N.; Papageorgiou, G. Z.; Floudas, G., Molecular Dynamics of Poly(ethylene-2,5-furanoate) (PEF) as a Function of the Degree of Crystallinity by Dielectric Spectroscopy and Calorimetry. Macromolecular Chemistry and Physics 2016, 217 (18), 20562062.

63. Bourdet, A.; Esposito, A.; Thiyagarajan, S.; Delbreilh, L.; Affouard, F.; Knoop, R. J. I.; Dargent, E., Molecular Mobility in Amorphous Biobased Poly(ethylene 2,5-furandicarboxylate) and Poly(ethylene 2,4-furandicarboxylate). Macromolecules 2018, 51 (5), 1937-1945.

64. Genovese, L.; Soccio, M.; Lotti, N.; Munari, A.; Szymczyk, A.; Paszkiewicz, S.; Linares, A.; Nogales, A.; Ezquerra, T. A., Effect of chemical structure on the subglass relaxation dynamics of biobased polyesters as revealed by dielectric spectroscopy: 2,5-furandicarboxylic acid vs. trans- 
1,4-cyclohexanedicarboxylic acid. Physical Chemistry Chemical Physics 2018, 20 (23), 1569615706.

65. Tobolsky, A. V., Properties and Structures of Polymers. Wiley: 1960.

66. Ferry, J. D., Viscoelastic Properties of Polymers. 3rd ed.; Wiley: 1980.

67. Williams, M. L.; Landel, R. F.; Ferry, J. D., The Temperature Dependence of Relaxation Mechanisms in Amorphous Polymers and Other Glass-forming Liquids. Journal of the American Chemical Society 1955, 77 (14), 3701-3707.

68. Rubinstein, M.; Colby, R. H., Polymer Physics. Oxford University Press: 2003.

69. Araujo, C. F.; Nolasco, M. M.; Ribeiro-Claro, P. J. A.; Rudić, S.; Silvestre, A. J. D.; Vaz, P. D.; Sousa, A. F., Inside PEF: Chain Conformation and Dynamics in Crystalline and Amorphous Domains. Macromolecules 2018, 51 (9), 3515-3526.

70. Soccio, M.; Nogales, A.; Lotti, N.; Munari, A.; Ezquerra, T. A., Evidence of Early Stage Precursors of Polymer Crystals by Dielectric Spectroscopy. Physical Review Letters 2007, 98 (3), 037801.

71. Soccio, M.; Nogales, A.; Lotti, N.; Munari, A.; Ezquerra, T. A., The $\beta$ relaxation as a probe to follow real-time polymer crystallization in model aliphatic polyesters. Polymer 2007, 48 (16), 4742-4750.

72. Soccio, M.; Nogales, A.; Martín-Fabiani, I.; Lotti, N.; Munari, A.; Ezquerra, T. A., Relaxation dynamics and cold crystallization of poly(pentamethylene terephthalate) as revealed by dielectric spectroscopy. Polymer 2014, 55 (6), 1552-1559. 


\section{Supporting Information}

Table S1. XRD reflections measured for Aged-PPeF.

\begin{tabular}{|c|c|c|}
\hline $\mathbf{2 \theta}$ & $\mathbf{q}\left(\mathbf{A}^{-\mathbf{1}}\right)$ & $\mathbf{d}(\mathbf{A})$ \\
\hline 10.72 & 0.76 & 8.24 \\
\hline 15.48 & 1.10 & 5.72 \\
\hline 17.38 & 1.23 & 5.10 \\
\hline 19.05 & 1.35 & 4.66 \\
\hline 20.49 & 1.45 & 4.33 \\
\hline 21.52 & 1.52 & 4.13 \\
\hline 23.40 & 1.65 & 3.80 \\
\hline 25.61 & 1.80 & 3.48 \\
\hline 28.65 & 2.01 & 3.11 \\
\hline
\end{tabular}

Figure S0. XRD peak deconvolution and peak position of Aged-PPeF sample.

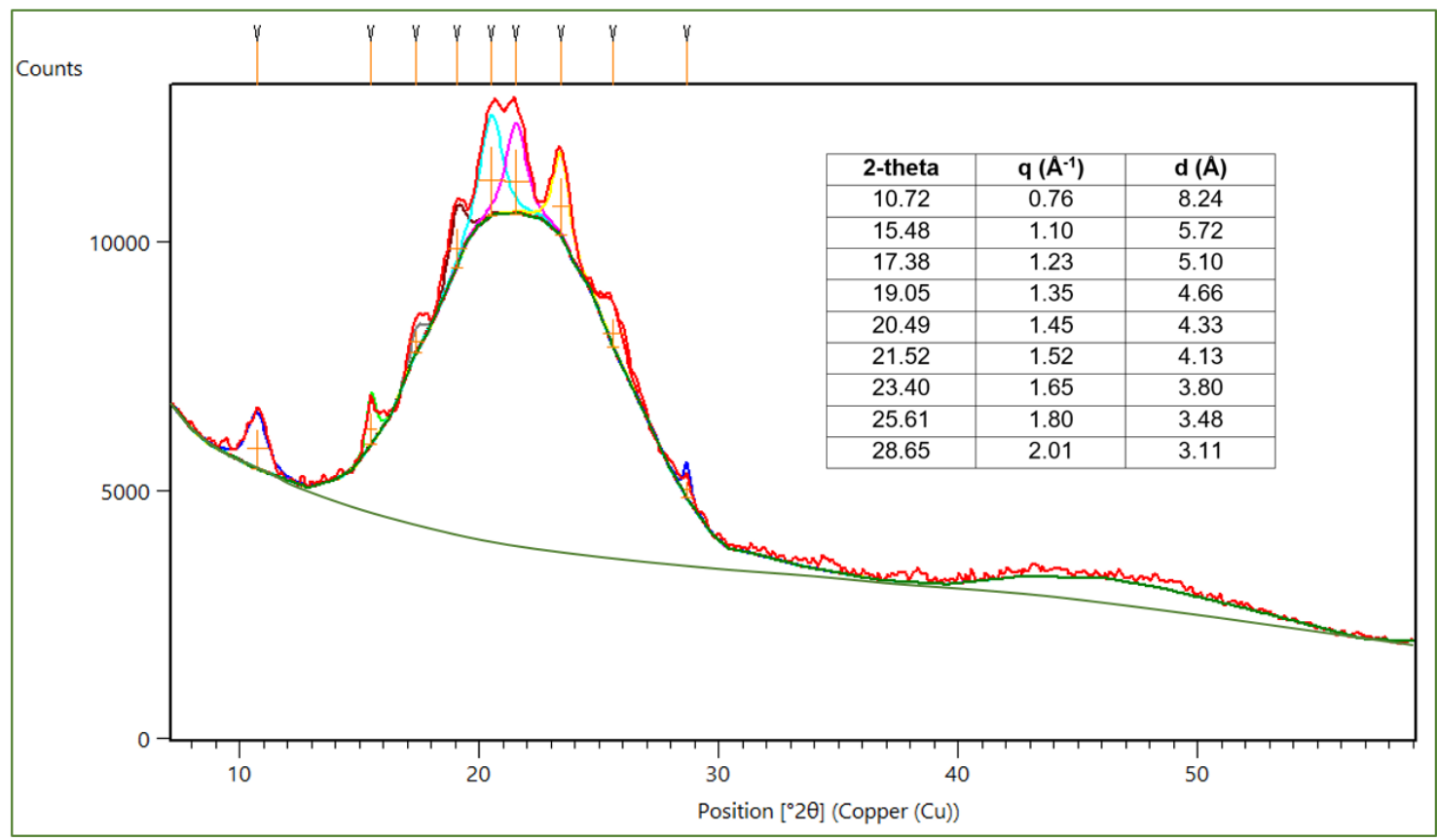


Figure S1. DSC (left) and XRD (right) scans of PPeF samples as a function of storage time.
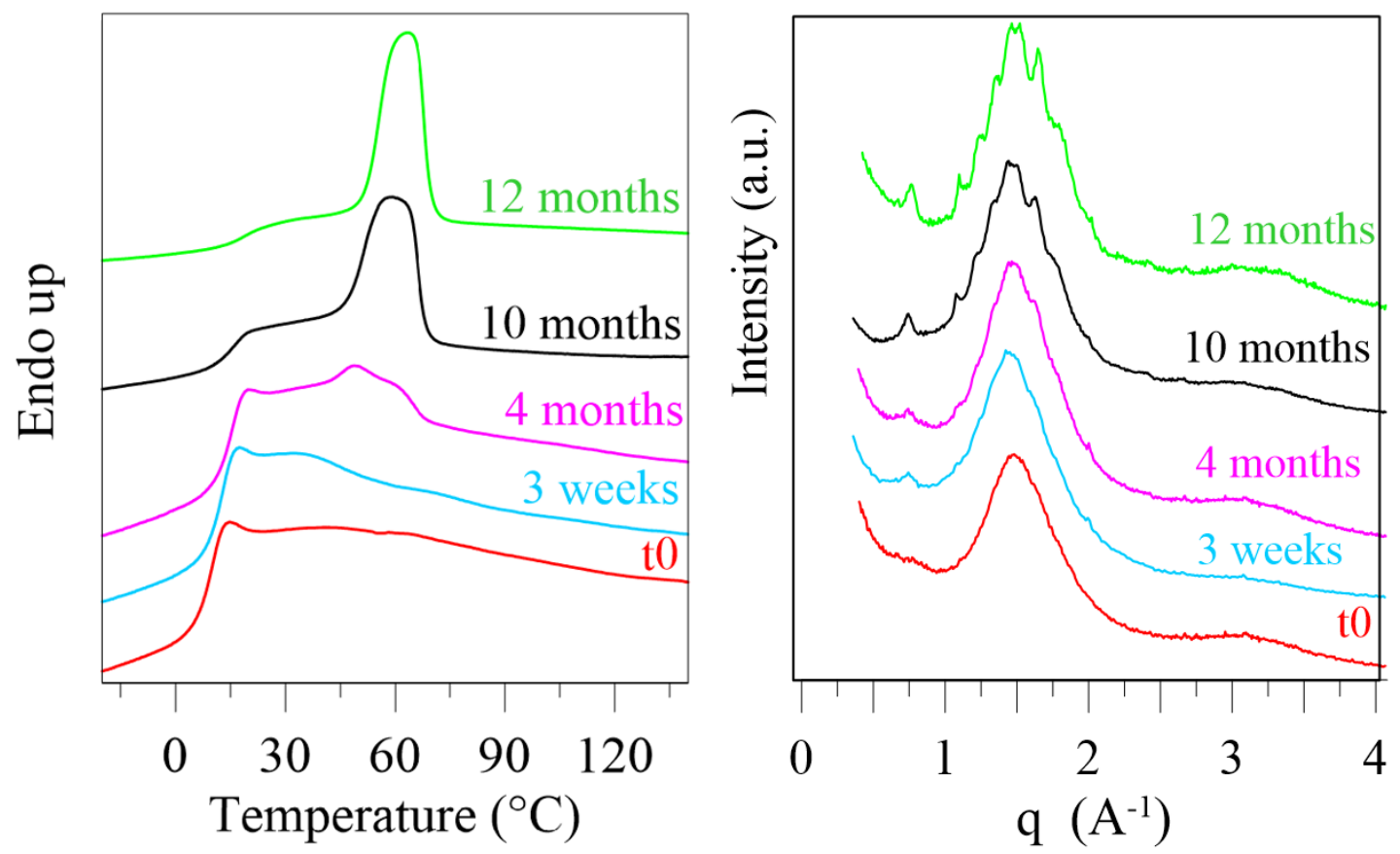

Figure S2. BDS data and fits at $243 \mathrm{~K}$ for (a) FC- PPeF, (b) SL-PPeF, (c) Aged-PPeF
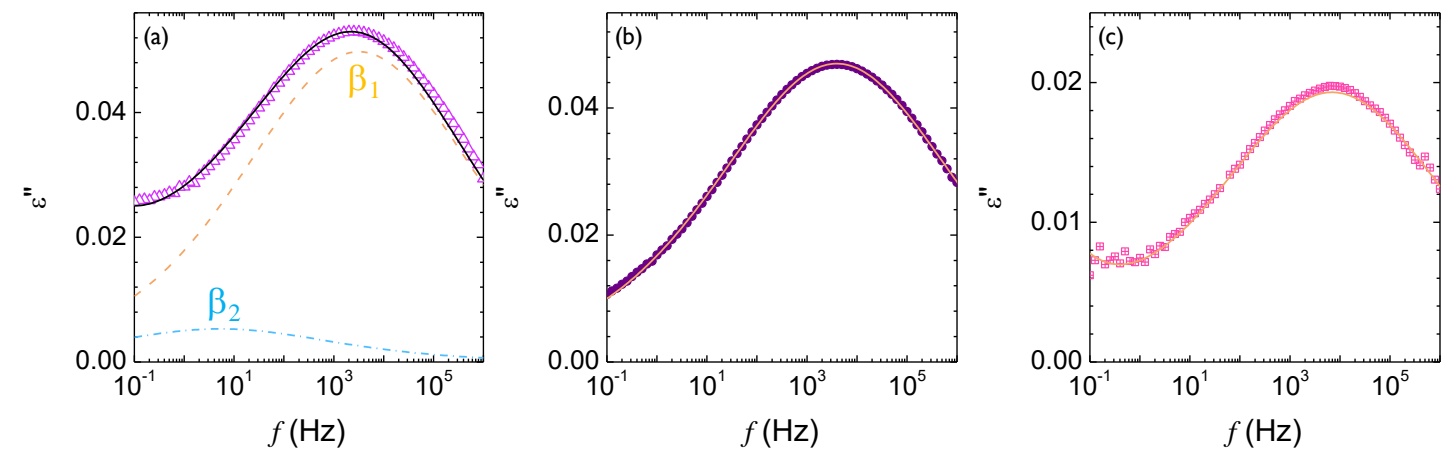

Figure S3. BDS data and fits at 303K, 333K, and 348K 

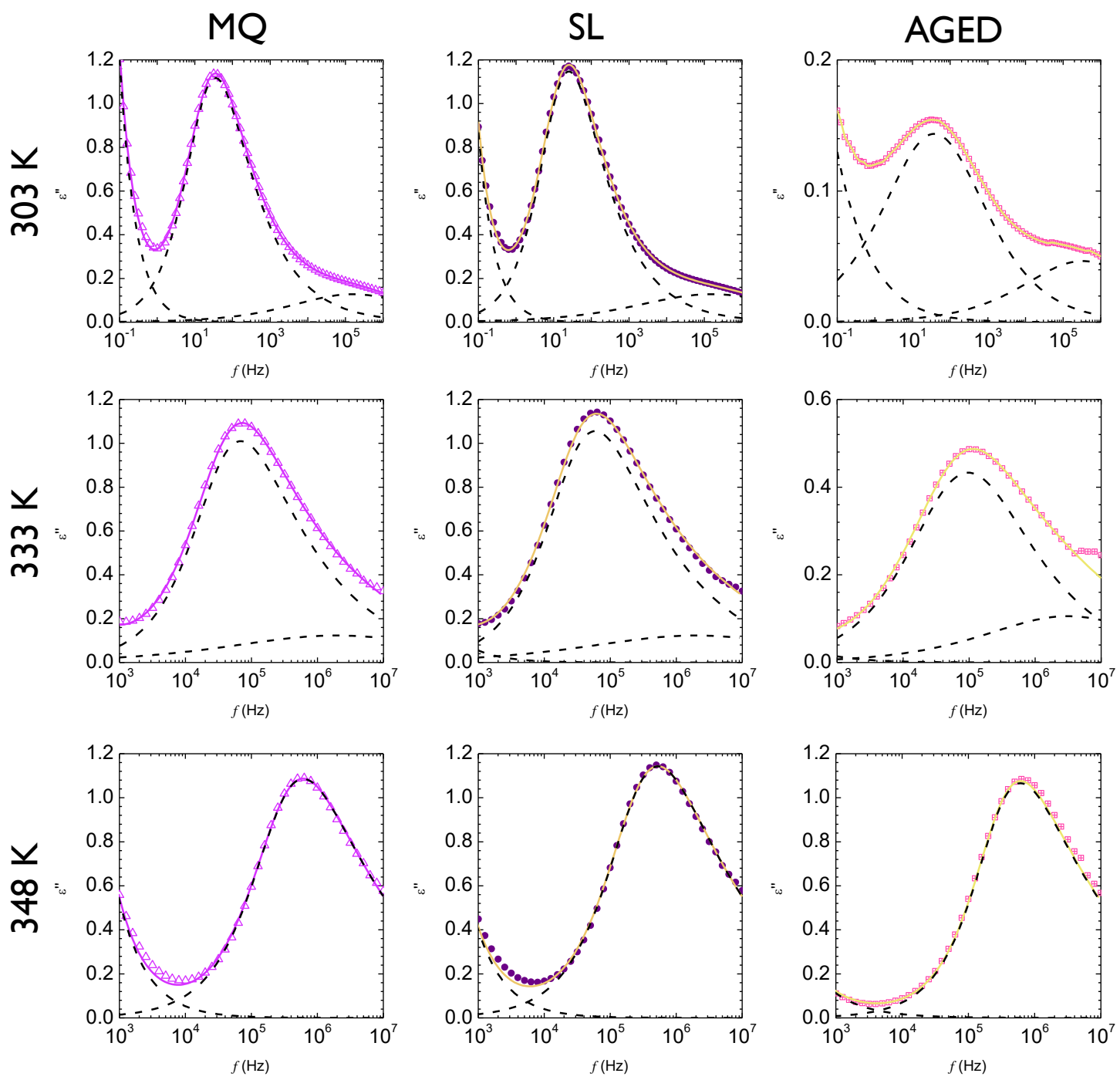

Figure S4. A simple physical model of the secondary relaxation is the assumption that the dipolar entities have to overcome an energy barrier separating equivalent positions and that the barriers are distributed following a Gaussian function (of standard deviation $\sigma_{\mathrm{E}}$ ). If the relaxation times follows the Arrhenius equation (Eq. 3 of the manuscript) the secondary relaxation process can be described as the superposition of Debye processes according to a Gaussian distribution of $\log _{10}(\tau)$ with a temperature dependent standard deviation $\sigma_{\mathrm{G}}(T) \sim \sigma_{\mathrm{E}} \frac{\log e}{R T}$. 
The symmetrical broadening of the corresponding $\mathrm{CC}$ fitting function, $b$ in eqn (1) in the main manuscript can be directly connected with the standard deviation $\sigma_{\mathrm{G}}$. To calculate this relationship, we generated $\varepsilon^{\prime \prime}(\omega)$ functions from the superposition of Debye processes according to a Gaussian distribution of $\log _{10}(\tau)$. The obtained curves were fitted to $\mathrm{CC}$ functions to obtain the $b$ parameter that corresponded to each $\sigma_{\mathrm{G}}$ (circles in Figure S4). The continuous line in Figure S4 is the best fit to eqn (4) in the main manuscript.

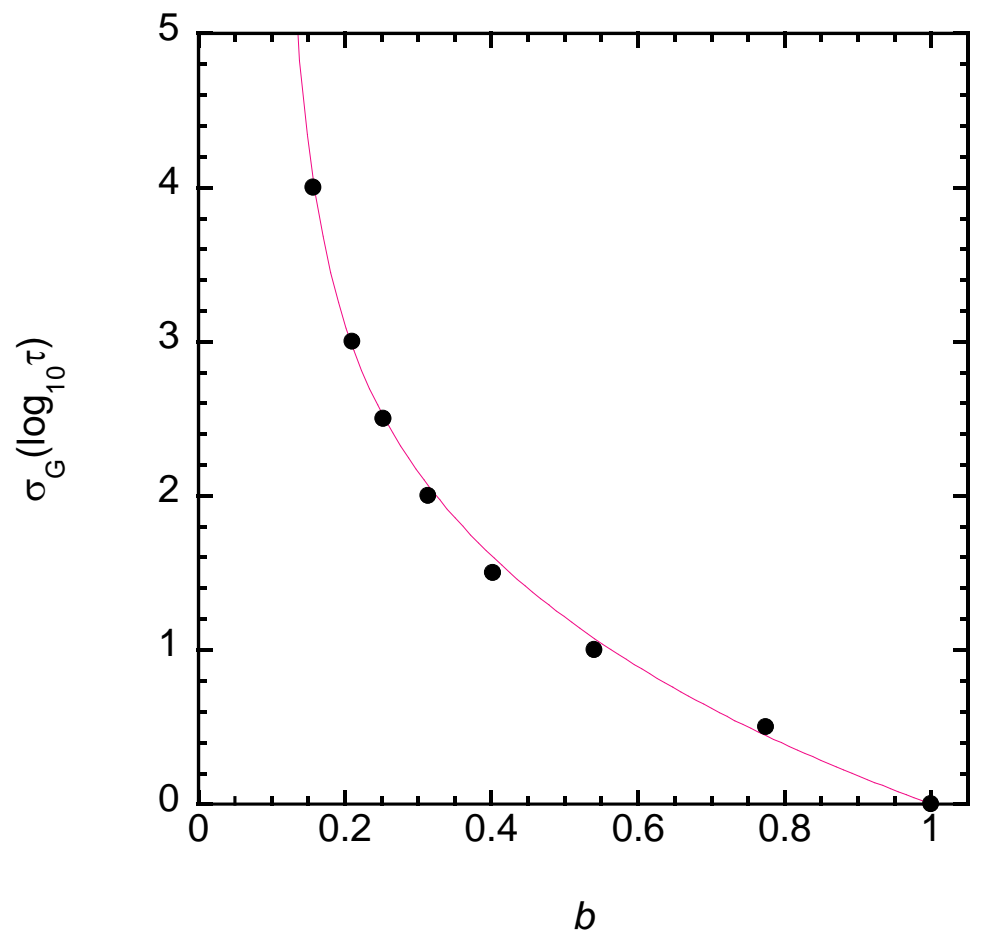


Figure S5. Schematic representation of the thermal treatment used to evaluate the evolution of the local relaxation below the glass transition temperature.

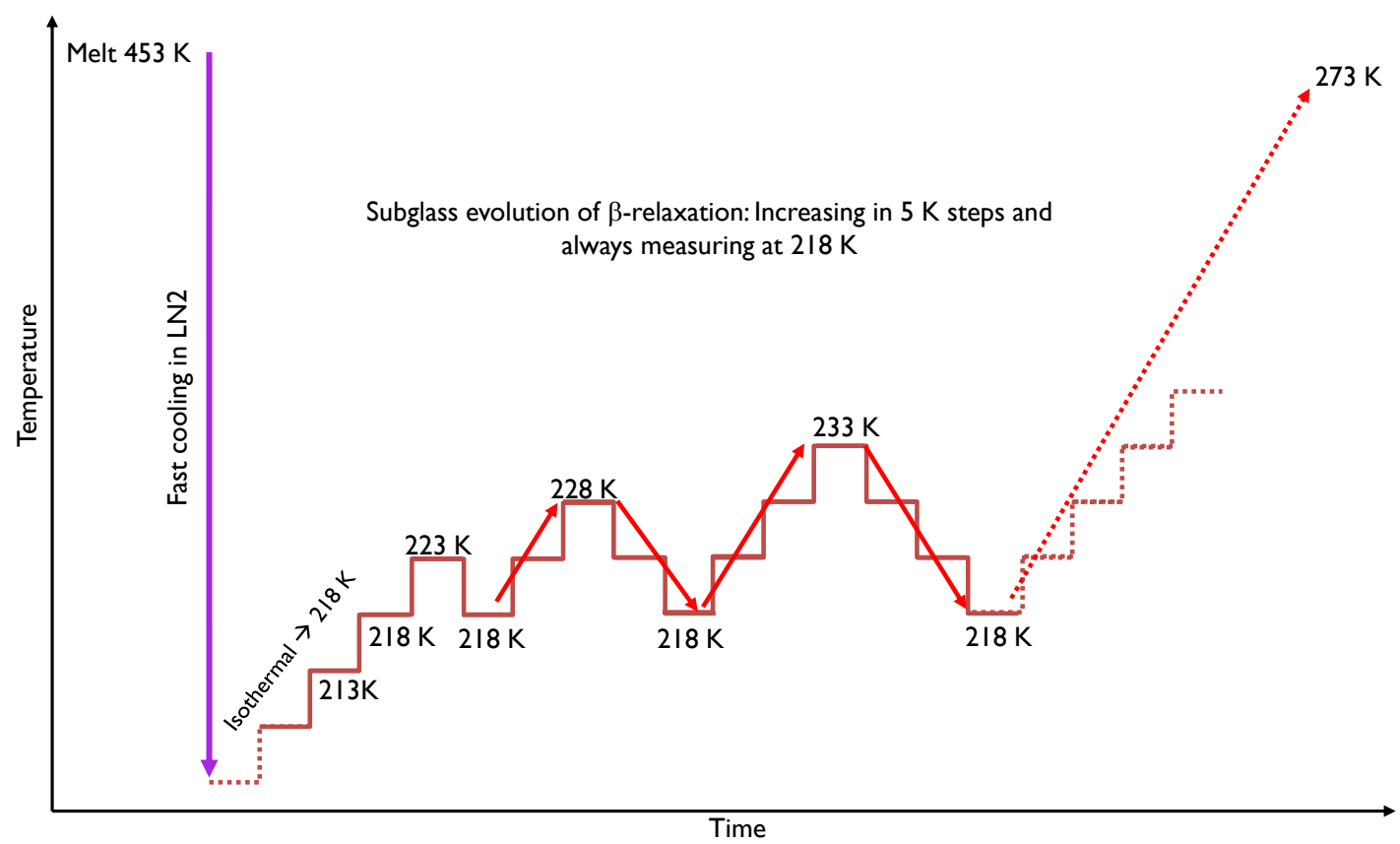


Figure S6. Dielectric loss spectra at (a) $303 \mathrm{~K}$, (b) $333 \mathrm{~K}$, and (c) $348 \mathrm{~K}$ for a PPeF capacitor aged for 3 months before melting (solid stars), the same sample after fast cooled (empty triangles) and slow cooled from the melt (solid circles).
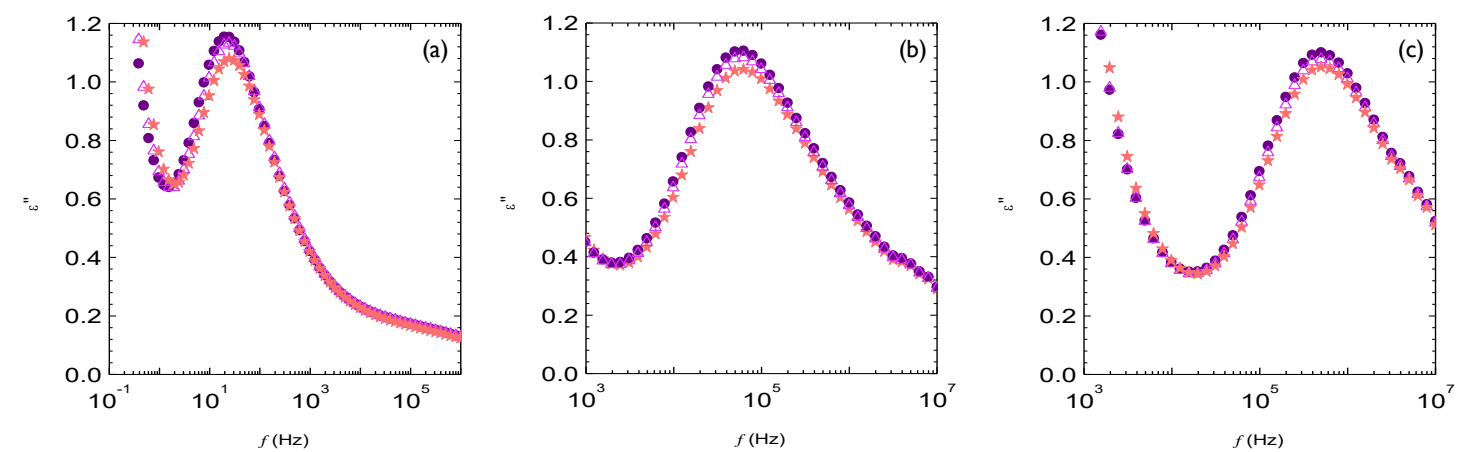